\title{
Generalized photon-added associated hypergeometric coherent states: characterization and relevant properties
}

\author{
K Sodoga $\ddagger^{a, b}$, I. Aremua ${ }^{a, b} \S$ and M N Hounkonnou ${ }^{b} \dagger$ \\ ${ }^{a}$ Université de Lomé Faculté des Sciences, Département de Physique, \\ Laboratoire de Physique des Matériaux et de Mécanique Appliquée, \\ 02 BP 1515 Lomé, Togo \\ ${ }^{b}$ International Chair in Mathematical Physics and Applications (ICMPA-UNESCO \\ Chair), University of Abomey-Calavi, 072 B.P. 050 Cotonou, Benin
}

\begin{abstract}
This paper presents the construction of a new set of generalized photonadded coherent states related to associated hypergeometric functions introduced in our previous work (Hounkonnou M N and Sodoga K 2005 J. Phys. A: Math. Gen 38 7851). These states satisfy all required mathematical and physical properties. The associated Stieltjes power-moment problem is explicitly solved by using Meijer's G-function and the Mellin inversion theorem. Relevant quantum optical and thermal characteristics are investigated. The formalism is applied to particular cases of the associated Hermite, Laguerre, Jacobi polynomials and hypergeometric functions. Their corresponding states exhibit sub-Poissonian photon number statistics.
\end{abstract}

$\ddagger$ ksodoga@tg.refer.org

$\S$ claudisak@yahoo.fr

$\dagger$ To whom correspondence should be addressed: norbert.hounkonnou@cipma.uac.bj, with copy to hounkonnou@yahoo.fr 


\section{Introduction}

Coherent states (CS) attracted much attention in the recent decades since their introduction by Schrödinger in 1926 [1] in the context of the one-dimensional harmonic oscilator, as the Heisenberg minimum-uncertainty states. Later they were rediscovered by Klauder, Glauber, and Sudarshan at the beginning of the 1960s [2]-44. Since then, CS and their various generalizations [5]-[13] are spread in the literature on quantum physics, atomic and condensed matter physics [10, 11, as well as in quantization problems [14]-16] (and references listed therein) with related mathematical tools [8]. For more details on the CS construction and their various generalizations, see also [17, 18]. CS defines an overcomplete family of vectors in the Hilbert space describing a physical problem. The canonical CS can be generalized as follows. Let $\left\{\phi_{m}\right\}_{m=0}^{\infty}$ be an orthonormal basis of a separable Hilbert space $\mathfrak{H}$, and $\mathfrak{D}$ an open subset of $\mathbb{C}$. For $z \in \mathfrak{D}$, with $z=r e^{i \theta}$, the CS are defined as

$$
|z\rangle=\left[\mathcal{N}\left(|z|^{2}\right)\right]^{-\frac{1}{2}} \sum_{m=0}^{\infty} \frac{z^{m}}{\sqrt{\rho(m)}} \phi_{m} \in \mathfrak{H}
$$

where $\{\rho(m)\}$ is a positive sequence of real numbers and $\mathcal{N}\left(|z|^{2}\right)$ is the normalization constant. As stated by Klauder [3], 10], the states $|z\rangle$, in order to be considered as CS, need to satisfy the following set of minimal criteria:

(a) Normalizability, i. e., $\langle z \mid z\rangle=1$

(b) Continuity in label $z$, i. e., $\left|z-z^{\prime}\right| \rightarrow 0 \Longrightarrow|\| z\rangle-\left|z^{\prime}\right\rangle|| \rightarrow 0$

(c) Completeness, i. e., there exists a positive weight fonction $\omega\left(|z|^{2}\right)$ such that

$$
\iint_{\mathbb{C}} d^{2} z|z\rangle \omega\left(|z|^{2}\right)\langle z|=\mathbb{1} .
$$

Cotfas, in a recent work [19], provided a factorization method of associated hypergeometric operators, and deduced the associated algebra and corresponding CS. The latters are eigenstates of the annihilation operator denoted $a_{m}$. Following Aleixo et al [20], we introduced, in a previous paper [21, a right inverse operator $a_{m}^{-1}$ allowing the definition of generalized associated hypergeometric CS (GAH-CS).

In this paper, we present the construction of a new family of CS, hereafter called generalized photon-added associated hypergeometric CS (GPAH-CS), obtained by adding photons to the conventional associated hypergeometric CS. Since the work by Agarwal and Tara [22], the photon-added CS (PACS) are intensively studied. They are intermediate between a single-photon Fock state $|n\rangle$ and a coherent one $|z\rangle$. PACS have various applications in quantum optics, quantum information and computation. See [24]-[31] and references therein. They also can generate the entangled states [23].

In some previous works [27, 28, the relevant statistical properties and thermal expectation values were investigated and analyzed in the photon-added Barut-Girardello CS, the PACS and the generalized hypergeometric thermal CS representations. The two latters were obtained from the definition of Appl and Schiller [32], for the pseudoharmonic oscillator and the Morse one-dimensional Hamiltonian, respectively. In one of our previous 
papers [29], a family of photon added as well as photon depleted CS related to the inverse of ladder operators acting on hypergeometric CS [32], was introduced. Their squeezing and antibunching properties were investigated in both standard (nondeformed) and deformed quantum optics. Recently, a new class of generalized PACS was constructed by excitations on a family of generalized CS. Their non-classical features and their quantum statistical properties were compared with the results obtained by Agarwals PACS [30].

The paper is organized as follows: first, in Section 2, we give a brief review on the Cotfa's factorization method of the hypergeometric operators, the algebra generated by the corresponding lowering and raising operators, and the construction of the GAHCS. Then, in Section 3, we generate the GPAH-CS by successive applications of the raising operator on the conventional GAH-CS. The inner product of two different GPAHCS is nonzero, highlighting that the obtained states are not mutually orthogonal. The associated Stieltjes power-moment problem is explicitly solved by using Meijer's Gfunction and the Mellin inversion theorem. The reproducing kernels in these GPAH-CS are provided. Some thermal statistical properties as well as the photon number statistics of the GPAH-CS in terms of the Mandel Q-parameter and the second-order correlation function, are computed and discussed. Next, in Section 4, the case of associated Hermite, Laguerre, Jacobi polynomials and hypergeometric functions is considered. Finally, we conclude in Section 5 with a summary of our main results.

\section{Generalized associated hypergeometric coherent states}

We start with the following definition.

Definition 2.1 The generalized associated hypergeometric type $C S$ (GAH-CS) are the CS corresponding to the $m^{\text {th }}$ derivative $\Phi_{l, m}=\kappa^{m} \Phi_{l}^{(m)}$ of the classical orthogonal polynomials $\Phi_{l}$ satisfying the second order differential equation of hypergeometric type:

$$
\sigma(s) \Phi_{l}^{\prime \prime}(s)+\tau(s) \Phi_{l}^{\prime}(s)+\lambda_{l} \Phi_{l}(s)=0
$$

where $\lambda_{l}=-\frac{1}{2} l(l-1) \sigma^{\prime \prime}-l \tau^{\prime}, \kappa=\sqrt{\sigma}$, with $\sigma$ a nonnegative function; $\sigma$ and $\tau$ are polynomials of at most second and exactly first degrees, respectively.

The $\Phi_{l, m}$, called associated hypergeometric-type functions (AHF), are solutions of the eigenvalue problem $H_{m} \Phi_{l, m}=\lambda_{l} \Phi_{l, m}$ where the Hamiltonian operator $H_{m}$ is expressed as a second order differential operator as follows:

$$
H_{m}=-\sigma \frac{d^{2}}{d s^{2}}-\tau \frac{d}{d s}+\frac{m(m-2)}{4} \frac{\sigma^{\prime 2}}{\sigma}+\frac{m}{2} \tau \frac{\sigma^{\prime}}{\sigma}-\frac{1}{2} m(m-2) \sigma^{\prime \prime}-m \tau^{\prime} .
$$

The $\Phi_{l, m}$ are orthogonal

$$
\int_{a}^{b} \Phi_{l, m} \Phi_{k, m} \rho \mathrm{d} s=0, \quad l \neq k, \quad l, k \in\{m, m+1, m+2, \ldots\}
$$

with respect to the positive weight function $\rho$ related to the polynomial functions $\sigma$ and $\tau$ by the Pearson's equation $(\sigma \rho)^{\prime}=\tau \rho$, over the interval $(a, b)$, which can be finite or infinite. The operator $H_{m}$ factorizes as

$$
H_{m}-\lambda_{m}=A_{m}^{\dagger} A_{m}, \quad H_{m+1}-\lambda_{m}=A_{m} A_{m}^{\dagger}
$$


and fulfills the intertwining relations

$$
H_{m} A_{m}^{\dagger}=A_{m}^{\dagger} H_{m+1} \quad \text { and } \quad A_{m} H_{m}=H_{m+1} A_{m}
$$

The mutually formal adjoint first-order differential operators

$$
A_{m}: \mathcal{H}_{m} \longrightarrow \mathcal{H}_{m+1} \quad \text { and } \quad A_{m}^{\dagger}: \mathcal{H}_{m+1} \longrightarrow \mathcal{H}_{m}
$$

are defined as [19]

$$
A_{m}=\kappa(s) \frac{\mathrm{d}}{\mathrm{d} s}-m \kappa^{\prime}(s) \quad \text { and } \quad A_{m}^{\dagger}=-\kappa(s) \frac{\mathrm{d}}{\mathrm{d} s}-\frac{\tau(s)}{\kappa(s)}-(m-1) \kappa^{\prime}(s) .
$$

$\mathcal{H}_{m}$ is the Hilbert space of $\left\{\Phi_{k, m}\right\}_{k \geq m}$, for $m \in \mathbb{N}$, with respect to the inner product (5). We restrict ourselves to the case when for each $m \in \mathbb{N}, \mathcal{H}_{m}$ is dense in the Hilbert space $\mathcal{H}=\left\{\varphi \in L^{2}(\rho(s) d s)\right\}$ where $L^{2}$ is the space of square integrable functions. The following shape invariance relations are satisfied

$$
A_{m} A_{m}^{\dagger}=A_{m+1}^{\dagger} A_{m+1}+r_{m+1}, \quad r_{m+1}=\lambda_{m+1}-\lambda_{m}=-m \sigma^{\prime \prime}-\tau^{\prime},
$$

where eigenvalues $\lambda_{l}$ and eigenfunctions $\Phi_{l, m}$ are:

$$
\lambda_{l}=\sum_{k=1}^{l} r_{k}, \quad \Phi_{l, m}=\frac{A_{m}^{\dagger}}{\lambda_{l}-\lambda_{m}} \frac{A_{m+1}^{\dagger}}{\lambda_{l}-\lambda_{m+1}} \cdots \frac{A_{l-2}^{\dagger}}{\lambda_{l}-\lambda_{l-2}} \frac{A_{l-1}^{\dagger}}{\lambda_{l}-\lambda_{l-1}} \Phi_{l, l}
$$

for all $l \in \mathbb{N}$ and $m \in\{0,1,2, \ldots, l-1\}, \Phi_{l, l}$ satisfying the relation $A_{l} \Phi_{l, l}=0$.

The annihilation and creation operators are defined as

$$
a_{m}, a_{m}^{\dagger}: \mathcal{H}_{m} \longrightarrow \mathcal{H}_{m}, \quad a_{m}=U_{m}^{\dagger} A_{m} \quad \text { and } \quad a_{m}^{\dagger}=A_{m}^{\dagger} U_{m}
$$

within the unitary operator

$$
U_{m}: \mathcal{H}_{m} \longrightarrow \mathcal{H}_{m}, \quad U_{m}|l . m\rangle=|l+1, m+1\rangle .
$$

The states $|l, m\rangle=\frac{\Phi_{l, m}}{\left\|\Phi_{l, m}\right\|}$ are defined for all $l \geq m$ and for each $m \in \mathbb{N}$. The mutually formal adjoint operators $a_{m}$ and $a_{m}^{\dagger}$ act on the state $|l, m\rangle$ as

$a_{m}|l, m\rangle=\sqrt{\lambda_{l}-\lambda_{m}}|l-1, m\rangle \quad$ and $\quad a_{m}^{\dagger}|l, m\rangle=\sqrt{\lambda_{l+1}-\lambda_{m}}|l+1, m\rangle, \quad l \geq m$,

and satisfy the commutation relations:

$$
\left[a_{m}, a_{m}^{\dagger}\right]=\mathcal{R}_{m}, \quad\left[a_{m}^{\dagger}, \mathcal{R}_{m}\right]=\sigma^{\prime \prime} a_{m}^{\dagger} \quad \text { and } \quad\left[a_{m}, \mathcal{R}_{m}\right]=-\sigma^{\prime \prime} a_{m}
$$

where $\mathcal{R}_{m}=-\sigma^{\prime \prime} N_{m}-\tau^{\prime}, N_{m}: \mathcal{H}_{m} \longrightarrow \mathcal{H}_{m}$ is the number operator defined as $N_{m} \Phi_{l, m}=l \Phi_{l, m}$. Remark that, when $\operatorname{deg} \sigma=1$, the algebra defined by the generators in (9) is isomorphic to the Heisenberg-Weyl algebra [19]. In addition to the commutation relations (9), we have

$$
A_{m} \mathcal{R}_{m}=\mathcal{R}_{m+1} A_{m},
$$

and the similarity transformation

$$
U_{m} \mathcal{R}_{m} U_{m}^{\dagger}=\mathcal{R}_{m+1}+\sigma^{\prime \prime}, \quad \text { for all } m \in \mathbb{N} .
$$


Setting for all $m \in \mathbb{N},|n\rangle=|m+n, m\rangle, e_{n}=\lambda_{m+n}-\lambda_{m}, \quad m \in \mathbb{N}$, we obtain $a_{m}|n\rangle=\sqrt{e_{n}}|n-1\rangle, \quad a_{m}^{\dagger}|n\rangle=\sqrt{e_{n+1}}|n+1\rangle, \quad\left(H_{m}-\lambda_{m}\right)|n\rangle=e_{n}|n\rangle$.

The CS for AHF were provided by Cotfas [19] as:

$$
|z\rangle=\mathcal{N}\left(|z|^{2}\right) \sum_{n=0}^{\infty} \frac{z^{n}}{\sqrt{\varepsilon_{n}}}|n\rangle \quad \mathcal{N}\left(|z|^{2}\right)=\left[\sum_{n=0}^{\infty} \frac{|z|^{2 n}}{\varepsilon_{n}}\right]^{-1 / 2}
$$

for any $z$ in the open $\operatorname{disc} \mathcal{C}(O, \mathcal{R})$ with centre $O$ and radius $\mathcal{R}=\lim \sup _{n \rightarrow \infty} \sqrt[n]{\varepsilon_{n}} \neq 0$ where $\varepsilon_{n}=\left\{\begin{array}{ll}1 & \text { if } n=0 \\ e_{1} e_{2} \cdots e_{n} & \text { if } n>0\end{array}\right.$. These CS are eigenstates of the annihilation operator $a_{m}$, i.e., $a_{m}|z\rangle=z|z\rangle$.

Introducing the right-inverse operators $A_{m}^{-1}, a_{m}^{-1}$, we showed in [21] that the CS (13) can be rewritten as

$$
|z\rangle=\mathcal{N}\left(|z|^{2}\right) \sum_{n=0}^{\infty}\left(z a_{m}^{-1}\right)^{n}|0\rangle
$$

generalized as

$$
\left|z ; \mathcal{R}_{m}\right\rangle=\sum_{n=0}^{\infty}\left(z \mathcal{R}_{m} a_{m}^{-1}\right)^{n}|0\rangle=\sum_{n=0}^{\infty} \frac{z^{n}}{h_{n}\left(\mathcal{R}_{m}\right)}|n\rangle
$$

where

$$
h_{n}\left(\mathcal{R}_{m}\right)=\frac{\sqrt{\varepsilon_{n}}}{\prod_{k=0}^{n-1}\left(\mathcal{R}_{m}+k \sigma^{\prime \prime}\right)} \text { for } n \geq 1 \quad \text { and } \quad h_{0}\left(\mathcal{R}_{m}\right)=1 .
$$

The states (15) are eigenstates of $a_{m}$,

$$
a_{m}\left|z ; \mathcal{R}_{m}\right\rangle=z\left(\mathcal{R}_{m}-\sigma^{\prime \prime}\right)\left|z ; \mathcal{R}_{m}\right\rangle,
$$

and satisfy the second order differential equation

$$
\left\{a_{m}-z\left(\mathcal{R}_{m}-\sigma^{\prime \prime}\right)\right\} \frac{d}{d z}\left|z ; \mathcal{R}_{m}\right\rangle=\left(\mathcal{R}_{m}-\sigma^{\prime \prime}\right)\left|z ; \mathcal{R}_{m}\right\rangle .
$$

Furthermore, we generalized the CS (15) as:

$$
\left|z ; \mathcal{R}_{m}\right\rangle=\sum_{n=0}^{\infty}\left(z f\left(\mathcal{R}_{m}\right) a_{m}^{-1}\right)^{n}|0\rangle=\sum_{n=0}^{\infty} \frac{z^{n}}{h_{n}\left(\mathcal{R}_{m}\right)}|n\rangle
$$

for any analytical function $f$, where

$$
h_{n}\left(\mathcal{R}_{m}\right)=\frac{\sqrt{\varepsilon_{n}}}{\prod_{k=0}^{n-1} f\left(\mathcal{R}_{m}+k \sigma^{\prime \prime}\right)} \text { for } n \geq 1 \quad \text { and } \quad h_{0}\left(\mathcal{R}_{m}\right)=1
$$

The CS (19) are eigenstates of $a_{m}$,

$$
a_{m}\left|z ; \mathcal{R}_{m}\right\rangle=z f\left(\mathcal{R}_{m}-\sigma^{\prime \prime}\right)\left|z ; \mathcal{R}_{m}\right\rangle,
$$


and satisfy the condition

$$
\left\{a_{m}-z f\left(\mathcal{R}_{m}-\sigma^{\prime \prime}\right)\right\} \frac{d}{d z}\left|z ; \mathcal{R}_{m}\right\rangle=f\left(\mathcal{R}_{m}-\sigma^{\prime \prime}\right)\left|z ; \mathcal{R}_{m}\right\rangle
$$

Taking into account the fact that $\mathcal{R}_{m}$ is an operator which acts on the states $|n\rangle$ as

$$
\mathcal{R}_{m}|n\rangle=\left[-(m+n) \sigma^{\prime \prime}-\tau^{\prime}\right]|n\rangle=r_{m+n+1}|n\rangle,
$$

we rewrite the CS (19) under the form:

$$
|z ; m\rangle=\sum_{n=0}^{\infty} \frac{z^{n}}{h_{n}(m)}|n\rangle,
$$

where

$$
h_{n}(m)=\frac{\sqrt{\varepsilon_{n}}}{\prod_{k=0}^{n-1} f\left(r_{m+n+1-k}\right)} \text { for } n \geq 1 \text { and } h_{0}(m)=1 .
$$

The properties (21) and (22) become,

$$
\begin{aligned}
& a_{m}|z ; m\rangle=z f\left(r_{m+n+2}^{\prime \prime}\right)|z ; m\rangle \\
& \left\{a_{m}-z f\left(r_{m+n+2}\right)\right\} \frac{d}{d z}|z ; m\rangle=f\left(r_{m+n+2}\right)|z ; m\rangle,
\end{aligned}
$$

respectively. We proved in [21] that the generalized coherent states (24) verify the properties of label continuity, overcompleteness, temporal stability and action identity.

\section{Construction of generalized photon-added associated hypergeometric coherent states}

In this section, we construct the generalized photon-added associated hypergeometric type coherent states (GPAH-CS) by repeating the action of the raising operator $a_{m}^{+}$on the GAH-CS.

Let $\mathfrak{H}_{m, p}$ be the Hilbert subspace of $\mathcal{H}_{m}$ defined as:

$$
\mathfrak{H}_{m, p}:=\operatorname{span}\{|n+p\rangle\}_{n, p \geq 0} .
$$

Let us note that the first $p$ number states $|n\rangle, n=0,1, \ldots, p-1$, are missing from the state $|z, m\rangle_{p} \in \mathfrak{H}_{m, p}$ defined below. Then, the unity operator in this subspace is to be written as 25

$$
\sum_{n=0}^{\infty}|n+p\rangle\langle n+p|=\mathbb{1}_{\mathfrak{H}_{m, p}}
$$

where $\mathbb{1}_{\mathfrak{H}_{m, p}}$ is the identity operator on $\mathfrak{H}_{m, p}$. Recall that $\mathbb{1}_{\mathfrak{H}_{m, p}}$ is only required to be a bounded positive operator with a densely defined inverse [9].

The GPAH-CS, denoted by $|z, m\rangle_{p}$, are defined as :

$$
|z, m\rangle_{p} \equiv\left(a_{m}^{+}\right)^{p}|z, m\rangle \text {. }
$$


where $p$ is a positive integer standing for the number of added quanta (or photons). From (9), we obtain

$$
a_{m}^{+} \mathcal{R}_{m}=\left(\mathcal{R}_{m}+\sigma^{\prime \prime}\right) a_{m}^{+}, \quad a_{m}^{+} f\left(\mathcal{R}_{m}\right)=f\left(\mathcal{R}_{m}+\sigma^{\prime \prime}\right) a_{m}^{+},
$$

for any analytical function $f$.

The GAH-CS (19) can be written as

$$
\left|z, \mathcal{R}_{m}\right\rangle=|0\rangle+\sum_{n=1}^{\infty} \frac{z^{n}}{\sqrt{\varepsilon_{n}}}\left(\prod_{k=0}^{n-1} f\left(\mathcal{R}_{m}+k \sigma^{\prime \prime}\right)\right)|n\rangle,
$$

and using Eqs (12) and (31) we obtain

$$
a_{m}^{+}\left|z, \mathcal{R}_{m}\right\rangle=\sqrt{e_{1}}|1\rangle+\sum_{n=1}^{\infty} z^{n} \frac{\sqrt{e_{n+1}}}{\sqrt{\varepsilon_{n}}}\left(\prod_{k=1}^{n} f\left(\mathcal{R}_{m}+k \sigma^{\prime \prime}\right)\right)|n+1\rangle .
$$

Now, applying $p$-times the operator $a_{m}^{+}$on the state $\left|z, \mathcal{R}_{m}\right\rangle$ leads to

$$
\left(a_{m}^{+}\right)^{p}\left|z, \mathcal{R}_{m}\right\rangle=\sqrt{\varepsilon_{p}}|p\rangle+\sum_{n=1}^{\infty} z^{n} \frac{\sqrt{e_{n+1} \cdot e_{n+2} \cdots e_{n+p}}}{\sqrt{\varepsilon_{n}}}\left(\prod_{k=p}^{n+p-1} f\left(\mathcal{R}_{m}+k \sigma^{\prime \prime}\right)\right)|n+p\rangle .
$$

The GPAH-CS can be rewritten in the form

$$
\left|z, \mathcal{R}_{m}\right\rangle_{p}=\sum_{n=0}^{\infty} \frac{z^{n}}{K_{n}^{p}\left(\mathcal{R}_{m}\right)}|n+p\rangle
$$

where the expansion coefficient is given by

$$
K_{n}^{p}\left(\mathcal{R}_{m}\right)=\frac{\varepsilon_{n}}{\sqrt{\varepsilon_{n+p}}} \frac{1}{\prod_{k=p}^{n+p-1} f\left(\mathcal{R}_{m}+k \sigma^{\prime \prime}\right)}, \quad \text { for } n \geq 1 \quad \text { and } \quad K_{0}^{p}\left(\mathcal{R}_{m}\right)=\frac{1}{\sqrt{\varepsilon_{p}}} .
$$

Taking into account Eq. (23), the GPAH-CS take the final form

$$
|z, m\rangle_{p}=\mathcal{N}_{p}\left(|z|^{2}, m\right) \sum_{n=0}^{\infty} \frac{z^{n}}{K_{n}^{p}(m)}|n+p\rangle
$$

where $\mathcal{N}_{p}\left(|z|^{2}, m\right)$ is the normalization constant evaluated below while the expansion coefficient is given by

$$
K_{n}^{p}(m)=\frac{\varepsilon_{n}}{\sqrt{\varepsilon_{n+p}}} \frac{1}{\prod_{k=0}^{n-1} f\left(r_{m+n+1-k}\right)} \quad \text { for } n \geq 1 \quad \text { and } \quad K_{0}^{p}(m)=\frac{1}{\sqrt{\varepsilon_{p}}} .
$$

In the particular case where $\operatorname{deg} \sigma=1, r_{m+n+1-k}=-\tau^{\prime}=$ constant $=c$. Then, the expansion coefficient takes the form

$K_{n}^{p}\left(\mathcal{R}_{m}\right)=\frac{\varepsilon_{n}}{\sqrt{\varepsilon_{n+p}}} \frac{1}{[f(c)]^{n}} \quad$ for $\quad n \geq 1 \quad$ and $\quad K_{0}^{p}\left(\mathcal{R}_{m}\right)=\frac{1}{\sqrt{\varepsilon_{p}}}$.

Let us examine the realization of the properties of normalization, label continuity and overcompleteness for the GPAH-CS (36) in the following subsections. 


\subsection{Normalization and non-orthogonality}

The normalization of the GPAH-CS (36) is obtained by requiring ${ }_{p}\langle z, m \mid z, m\rangle_{p}=1$. A straightforward computation gives the normalization constant $\mathcal{N}_{p}\left(|z|^{2} ; m\right)$ as:

$$
\mathcal{N}_{p}\left(|z|^{2} ; m\right)=\left[\sum_{n=0}^{\infty} \frac{|z|^{2 n}}{\left|K_{n}^{p}(m)\right|^{2}}\right]^{-1 / 2} .
$$

The inner product of two different states $|z ; m\rangle_{p}$ and $\left|z^{\prime} ; m\right\rangle_{p^{\prime}}$ reads as:

$p^{\prime}\left\langle z^{\prime} ; m \mid z ; m\right\rangle_{p}=\mathcal{N}_{p^{\prime}}\left(\left|z^{\prime}\right|^{2} ; m\right) \mathcal{N}_{p}\left(|z|^{2} ; m\right) \sum_{n, n^{\prime}=0}^{\infty} \frac{z^{\prime \star n^{\prime}} z^{n}}{K_{n^{\prime}}^{p^{\prime \star}}(m) K_{n}^{p}(m)}\left\langle n^{\prime}+p^{\prime} \mid n+p\right\rangle$.

Due to the orthonormality of the states $|n\rangle$, the inner product (40) gives

${ }_{p^{\prime}}\left\langle z^{\prime} ; m \mid z ; m\right\rangle_{p}=\mathcal{N}_{p^{\prime}}\left(\left|z^{\prime}\right|^{2} ; m\right) \mathcal{N}_{p}\left(|z|^{2} ; m\right) z^{\prime \star\left(p-p^{\prime}\right)} \sum_{n=0}^{\infty} \frac{\left(z^{\prime \star} z\right)^{n}}{{K_{n+p-p^{\prime}}^{p^{\prime}}}^{\star}(m) K_{n}^{p}(m)}$

proving that the GPAH-CS are not mutually orthogonal.

\subsection{Label continuity}

In the Hilbert space $\mathcal{H}_{m}$, the GPAH-CS $|z, m\rangle_{p}$ are labeled by $p$ and $z$. The label continuity condition can then be stated as:

$\left|z-z^{\prime}\right| \rightarrow 0$ and $\left|p-p^{\prime}\right| \rightarrow 0 \Longrightarrow|||z, m\rangle_{p}-\left|z^{\prime}, m\right\rangle_{p^{\prime}} \|^{2}=2\left[1-\mathcal{R} e\left({ }_{p^{\prime}}\left\langle z^{\prime} ; m \mid z ; m\right\rangle_{p}\right)\right] \rightarrow 0$.

This is satisfied by the states $|z, m\rangle_{p}$, since from Eqs. (39, 41), we see that

$$
p \rightarrow p^{\prime} \quad \text { and } \quad z \rightarrow z^{\prime} \Longrightarrow{ }_{p^{\prime}}\left\langle z^{\prime} ; m \mid z ; m\right\rangle_{p} \rightarrow 1 \text {. }
$$

Therefore the GPAH-CS $|z, m\rangle_{p}$ are continuous in their labels.

\subsection{Overcompleteness}

We have to determine a non-negative weight function $\omega_{p}\left(|z|^{2} ; m\right)$ such that the overcompleteness or the resolution of identity

$$
\int_{\mathbb{C}} d^{2} z|z ; m\rangle_{p} \omega_{p}\left(|z|^{2} ; m\right)_{p}\left\langle z ; m\left|=\mathbb{1}_{\mathfrak{H}_{m, p}} \equiv \sum_{n=0}^{\infty}\right| n+p\right\rangle\langle n+p|
$$

holds.

Taking to account the definition (36) of the GPACS, we have:

$$
\int_{\mathbb{C}} d^{2} z \mathcal{N}_{p}^{2}\left(|z|^{2} ; m\right) \sum_{n, n^{\prime}=0}^{\infty} \frac{z^{\star n^{\prime}} z^{n}}{K_{n^{\prime}}^{p \star}(m) K_{n}^{p}(m)}\left|n^{\prime}+p\right\rangle\langle n+p| \omega_{p}\left(|z|^{2} ; m\right)=\mathbb{1}_{\mathfrak{H}_{m, p}} .
$$

The diagonal matrix elements of the above relation, using the orthonormality of the number states $|n\rangle$, gives:

$$
\int_{\mathbb{C}} d^{2} z \mathcal{N}_{p}^{2}\left(|z|^{2} ; m\right)|z|^{2 n} \omega_{p}\left(|z|^{2} ; m\right)=\left|K_{n}^{p}(m)\right|^{2}
$$


This equation, after performing the angular integration, gives:

$\int_{0}^{\infty} d x x^{n} \mathcal{W}_{p}(x ; m)=\left|K_{n}^{p}(m)\right|^{2}, \quad$ with $\quad \mathcal{W}_{p}(x ; m)=\pi \mathcal{N}_{p}^{2}(x ; m) \omega_{p}(x ; m)$

where we use the polar representation $z=r e^{i \phi} ; x$ standing for $|z|^{2}=r^{2}$. The weight function $\omega_{p}$ is then related to the undetermined moment distribution $\mathcal{W}_{p}(x ; m)$, which is the solution of the Stieltjes moment problem with the moments given by $\left|K_{n}^{p}(m)\right|^{2}$.

As pointed out in [26], the measure can be determined by using the Mellin transformation procedure. Let us rewrite (47) as

$\int_{0}^{\infty} d x x^{n+p} g_{p}(x ; m)=\left|K_{n}^{p}(m)\right|^{2}$, where $g_{p}(x ; m)=\pi \mathcal{N}_{p}^{2}(x ; m) x^{-p} \omega_{p}(x ; m)$.

Performing the variable change $n+p \rightarrow s-1$, Eq. (48) becomes:

$$
\int_{0}^{\infty} d x x^{s-1} g_{p}(x ; m)=\left|K_{s}^{p}(m)\right|^{2} .
$$

Consider the Meijer's G-function and the Mellin inversion theorem [38], [39]

$$
\int_{0}^{\infty} d x x^{s-1} G_{p, q}^{m, n}\left(\alpha x \mid \begin{array}{l}
a_{1}, \ldots, a_{n} ; a_{n+1}, \ldots, a_{p} \\
b_{1}, \ldots, b_{m} ; b_{m+1}, \ldots, b_{q}
\end{array}\right)=\frac{1}{\alpha^{s}} \frac{\prod_{j=1}^{m} \Gamma\left(b_{j}+s\right) \prod_{j=1}^{n} \Gamma\left(1-a_{j}-s\right)}{\prod_{j=m+1}^{q} \Gamma\left(1-b_{j}-s\right) \prod_{j=n+1}^{p} \Gamma\left(a_{j}+s\right)} .
$$

In the different examples of the next section, $\left|K_{s}^{p}(m)\right|^{2}$ in the above relation can be expressed in terms of Gamma functions as in the second member of the Mellin inversion theorem (50). Then comparing the equations (49) and (50), $g_{p}(x ; m)$ can be identified as the Meijer's G- function:

$$
g_{p}(x ; m)=G_{p, q}^{m, n}\left(\alpha x \mid \begin{array}{l}
a_{1}, \ldots, a_{n} ; a_{n+1}, \ldots, a_{p} \\
b_{1}, \ldots, b_{m} ; b_{m+1}, \ldots, b_{q}
\end{array}\right) .
$$

Once $g_{p}$ is determined, the measure $\omega_{p}$ can be deduced as

$$
\omega_{p}(x ; m)=\frac{x^{p}}{\pi} \frac{g_{p}(x ; m)}{\mathcal{N}_{p}^{2}(x ; m)} .
$$

The overcompleteness of the GPAH-CS on $\mathfrak{H}_{m, p}$ displayed in (44) and (45) brings us to discuss their relation with the reproducing kernels.

\subsection{Reproducing kernels}

Define the quantity $\mathcal{K}\left(z, z^{\prime}\right):={ }_{p}\left\langle z^{\prime} ; m \mid z ; m\right\rangle_{p}$. From

${ }_{p}\left\langle z^{\prime} ; m \mid z ; m\right\rangle_{p}=\mathcal{N}_{p}\left(\left|z^{\prime}\right|^{2} ; m\right) \mathcal{N}_{p}\left(|z|^{2} ; m\right) \sum_{n=0}^{\infty} \frac{\left(z^{\prime \star} z\right)^{n}}{\left|K_{n}^{p}(m)\right|^{2}}=\frac{\mathcal{N}_{p}\left(\left|z^{\prime}\right|^{2} ; m\right) \mathcal{N}_{p}\left(|z|^{2} ; m\right)}{\mathcal{N}_{p}^{2}\left(z^{\prime \star} z ; m\right)}$

we obtain

$\frac{{ }_{p}\left\langle z^{\prime} ; m \mid z ; m\right\rangle_{p}}{=}=\frac{\mathcal{N}_{p}\left(\left|z^{\prime}\right|^{2} ; m\right) \mathcal{N}_{p}\left(|z|^{2} ; m\right)}{\mathcal{N}_{p}^{2}\left(z^{\star} z^{\prime} ; m\right)}:=\mathcal{K}\left(z^{\prime}, z\right)$.

$\mathcal{K}\left(z, z^{\prime}\right)$ is a reproducing kernel through the following result: 
Proposition 3.1 The following properties

(i) hermiticity $\mathcal{K}\left(z, z^{\prime}\right)=\overline{\mathcal{K}\left(z^{\prime}, z\right)}$,

(ii) positivity $\mathcal{K}(z, z)>0$,

(iii) idempotence

$$
\int_{\mathbb{C}} d^{2} z^{\prime \prime} \omega_{p}\left(\left|z^{\prime \prime}\right|^{2} ; m\right) \mathcal{K}\left(z, z^{\prime \prime}\right) \mathcal{K}\left(z^{\prime \prime}, z^{\prime}\right)=\mathcal{K}\left(z, z^{\prime}\right)
$$

are satisfied by the function $\mathcal{K}$ on $\mathfrak{H}_{m}$.

Proof.

(i) Hermiticity: Using (53) and (54), we get

$$
\mathcal{K}\left(z, z^{\prime}\right)=\mathcal{K}\left(z^{\prime}, z\right)^{\star} \text {. }
$$

(ii) Positivity: From (54), we obtain

$$
\mathcal{K}(z, z)={ }_{p}\langle z ; m \mid z ; m\rangle_{p}=\frac{\mathcal{N}_{p}\left(|z|^{2} ; m\right) \mathcal{N}_{p}\left(|z|^{2} ; m\right)}{\mathcal{N}_{p}^{2}\left(|z|^{2} ; m\right)}=1>0 .
$$

(iii) Idempotence: Let $\mathcal{I}=\int_{\mathbb{C}} d^{2} z^{\prime \prime} \omega_{p}\left(\left|z^{\prime \prime}\right|^{2} ; m\right) \mathcal{K}\left(z, z^{\prime \prime}\right) \mathcal{K}\left(z^{\prime \prime}, z^{\prime}\right)$. Then, setting

$$
\begin{aligned}
& \xi_{p}\left(z, z^{\prime} ; m\right)=\mathcal{N}_{p}\left(|z|^{2} ; m\right) \mathcal{N}_{p}\left(\left|z^{\prime}\right|^{2} ; m\right) \text { gives } \\
& \mathcal{I}=\xi_{p}\left(z, z^{\prime} ; m\right) \int_{\mathbb{C}} d^{2} z^{\prime \prime} \omega_{p}\left(\left|z^{\prime \prime}\right|^{2} ; m\right) \frac{\mathcal{N}_{p}^{2}\left(\left|z^{\prime \prime}\right|^{2} ; m\right)}{\mathcal{N}_{p}^{2}\left(z z^{\prime \prime \star} ; m\right) \mathcal{N}_{p}^{2}\left(z^{\prime \prime} z^{\prime \star} ; m\right)} \\
& =\xi_{p}\left(z, z^{\prime} ; m\right) \sum_{k, l=0}^{\infty} \int_{0}^{\infty} \int_{0}^{2 \pi} \frac{e^{-i(k-l) \theta^{\prime \prime}} r^{\prime \prime k+l}}{\left|K_{k}^{p}(m)\right|^{2}} \frac{z^{k}\left(z^{\prime \star}\right)^{l}}{\left|K_{l}^{p}(m)\right|^{2}} \\
& =\xi_{p}\left(z, z^{\prime} ; m\right) r^{\prime \prime} d r^{\prime \prime} d \theta^{\prime \prime} \omega_{p}\left(\left|z^{\prime \prime}\right|^{2} ; m\right) \\
& =\xi_{p}\left(z, z^{\prime} ; m\right) \sum_{k=0}^{\infty} \frac{\left(\sqrt{z z^{\prime \star}}\right)^{2 k}}{\left|K_{k}^{m}(m)\right|^{2}}\left\{\int_{0}^{\infty} \frac{x^{\prime \prime k+m}}{\left.K_{k}^{m}(m)\right|^{2}} g_{m}\left(x^{\prime \prime} ; m\right) d x^{\prime \prime}\right\} \\
& =\frac{\mathcal{N}_{p}\left(|z|^{2} ; m\right) \mathcal{N}_{p}\left(\left|z^{\prime}\right|^{2} ; m\right)}{\mathcal{N}_{p}^{2}\left(z z^{\prime \star} ; m\right)}=\mathcal{K}\left(z, z^{\prime}\right)
\end{aligned}
$$

which completes the proof.

\subsection{Statistical properties}

This section is devoted to the investigation of some quantum optical features of the GPAH-CS, such as the Mandel Q-parameter, the second order correlation function, the photon number distribution (PND), the signal-to-quantum-noise ratio (SNR). Besides, the relevant thermal properties are discussed by considering a quantum gas of the system for which the density operator is elaborated. 


\subsubsection{Photon number statistics}

The photon number statistics can be first studied by means of the Mandel Q-parameter characterizing the Poisson distribution of photons, defined as [36]:

$$
Q=\frac{\left(\Delta N_{m}\right)^{2}}{\langle H\rangle}-1, \quad\left(\Delta N_{m}\right)^{2}=\left\langle N_{m}^{2}\right\rangle-\left\langle N_{m}\right\rangle^{2} .
$$

The Mandel Q-parameter determines whether the GPAH-CS have a photon number distribution. This latter is sub-Poissonian if $-1 \leq Q<0$, Poissoinian if $Q=0$, and super-Poissonian if $Q>0$.

Next, from the expression of the second order correlation function, we have

$$
g^{2}=\frac{\left\langle N_{m}^{2}\right\rangle-\left\langle N_{m}\right\rangle}{\left\langle N_{m}\right\rangle^{2}}
$$

where the mean values are

$$
\left\langle N_{m}\right\rangle={ }_{p}\left\langle z ; m\left|N_{m}\right| z ; m\right\rangle_{p}, \quad\left\langle N_{m}^{2}\right\rangle={ }_{p}\left\langle z ; p\left|N_{m}^{2}\right| z ; m\right\rangle_{p} .
$$

The second order correlation function determines either the bunching or anti-bunching

effects of the optical field: $g^{2}<1$ indicates that a photon is anti-bunching and thus the optical state is non-classical, while $g^{2} \geq 1$ defines the classical (or random) optical field. One can check that for a GPAH-CS (36), the expectation values of the number operator are provided as

$$
\begin{aligned}
& \left\langle N_{m}\right\rangle=\mathcal{N}_{p}^{2}\left(|z|^{2} ; m\right) \sum_{n=0}^{\infty}(m+n+p) \frac{|z|^{2 n}}{\left|K_{n}^{p}(m)\right|^{2}}, \\
& \left\langle N_{m}^{2}\right\rangle=\mathcal{N}_{p}^{2}\left(|z|^{2} ; m\right) \sum_{n=0}^{\infty}(m+n+p)^{2} \frac{|z|^{2 n}}{\left|K_{n}^{p}(m)\right|^{2}} .
\end{aligned}
$$

Another specific key useful for our purpose is the PND which represents an important tool for characterizing a given optical field (see for e.g, [37]). The PND exhibiting oscillations, which corresponds to the probability of finding $n$ quanta in the GPAH-CS, is given by the projection of $|z ; m\rangle_{p}$ in the state $|n\rangle$ as follows [24]

$$
\mathcal{P}_{n}^{p}(x ; m)=\left|\langle n \mid z ; m\rangle_{p}\right|^{2}=\mathcal{N}_{p}(x ; m)^{2} \frac{x^{n-p}}{\left|K_{n}^{p}(m)\right|^{2}}, \quad x=|z|^{2} .
$$

It reduces to a Poisson distribution for the conventional CS, for $p \rightarrow 0$.

Finally, we consider the SNR [24, 37] in the GPAH-CS, which is relevant when studying, for example, a nondeterministic, noiseless amplification of a CS in the context of use of photon addition and subtraction as a probabilistic amplifier and its effects on coherent light. When considering a CS as input and SNR as a metric, this latter is a remarkable tool used to enhance a general signal with no added noise. It improves as well as the effect of photon addition becames important. A general construction form for the SNR of a $p$ PACS is provided as follows [40]:

$$
S N R_{p}+=\frac{\left\langle N_{m}\right\rangle-p}{\Delta N_{m}}
$$


where $p$ is the number of added photons.

We will see in the examples of the next section that the coefficient $K_{n}^{p}(m)$ can be expressed in terms of Gamma functions. The normalized factor, the expectation values and the Mandel Q-parameter can be therefore written in terms of generalized hypergeometric functions ${ }_{p} F_{q}$.

\subsubsection{Thermal statistics}

In quantum mechanics, the important tool for characterizing the probability distribution on the states of a physical system is a statistical operator called density matrix, generally denoted by $\rho$. The latter is useful for examining the physical and chemical properties of a system (see for e.g. [27], [16] and references listed therein). Consider a quantum gas of the system in the thermodynamic equilibrium with a reservoir at temperature $T$, which satisfies a quantum canonical distribution. The corresponding normalized density operator is given as

$$
\rho^{(p)}=\frac{1}{Z} \sum_{n=0}^{\infty} e^{-\beta e_{n}}|n+p\rangle\langle n+p|
$$

where in the exponential $e_{n}$ is the eigen-energy, and the partition function $Z$ is taken as the normalization constant.

The diagonal elements of $\rho^{(p)}$ which are key ingredients for our purpose, also known as the $Q$-distribution or Husimi's distribution, are derived in the GPAH-CS basis as

$$
{ }_{p}\left\langle z ; m\left|\rho^{(p)}\right| z ; m\right\rangle_{p}=\frac{\mathcal{N}_{p}^{2}\left(|z|^{2} ; m\right)}{Z} \sum_{n=0}^{\infty} \frac{|z|^{2 n}}{\left|K_{n}^{p}(m)\right|^{2}} e^{-\beta e_{n}} .
$$

The normalization of the density operator leads to

$$
\operatorname{Tr} \rho^{(p)}=\int_{\mathbb{C}} d^{2} z \omega_{p}\left(|z|^{2} ; m\right)_{p}\left\langle z ; m\left|\rho^{(p)}\right| z ; m\right\rangle_{p}=1 .
$$

The diagonal expansion of the normalized canonical density operator over the GPAH-CS projector is

$$
\rho^{(p)}=\int_{\mathbb{C}} d^{2} z \omega_{p}\left(|z|^{2} ; m\right)|z ; m\rangle_{p} P\left(|z|^{2}\right)_{p}\langle z ; m|
$$

where the $P$-distribution function $P\left(|z|^{2}\right) \mid$ satisfying the normalization to unity condition

$$
\int_{\mathbb{C}} d^{2} z \omega_{p}\left(|z|^{2} ; m\right) P\left(|z|^{2}\right)=1
$$

must be determined.

Thus, given an observable $\mathcal{O}$, one obtains the expectation value, i. e., the thermal average given by

$$
\langle\mathcal{O}\rangle_{p}=\operatorname{Tr}\left(\rho^{(p)} \mathcal{O}\right)=\int_{\mathbb{C}} d^{2} z \omega_{p}\left(|z|^{2} ; m\right) P\left(|z|^{2}\right)_{p}\langle z ; m|\mathcal{O}| z ; m\rangle_{p}
$$

Using (62) and (63) together, the pseudo-thermal expectation value of the number operator $N_{m}$, and of its square $N_{m}^{2}$, given by $\left\langle N_{m}\right\rangle^{(p)}=\operatorname{Tr}\left(\rho^{(p)} N_{m}\right)$ and $\left\langle N_{m}^{2}\right\rangle^{(p)}=$ 
$\operatorname{Tr}\left(\rho^{(p)} N_{m}^{2}\right)$, respectively, allow to obtain the thermal intensity correlation function as follows:

$$
\left(g^{2}\right)^{(p)}=\frac{\left\langle N_{m}^{2}\right\rangle^{(p)}-\left\langle N_{m}\right\rangle^{(p)}}{\left(\left\langle N_{m}\right\rangle^{(p)}\right)^{2}} .
$$

Then, the thermal analogue of the Mandel parameter, given by

$$
Q^{(p)}=\left\langle N_{m}\right\rangle^{(p)}\left[\left(g^{2}\right)^{(p)}-1\right]
$$

is deduced.

In the illustrated examples, given an appropriate function $K_{n}^{p}(m)$, this formalism will be applied to determine the concrete expressions for the relations (66) $-(\mathbf{7 3})$.

\section{GPAH-CS for classical orthogonal polynomials}

In this section, the Hermite, Laguerre, Jacobi polynomials and hypergeometric functions are considered for the construction of GPAH-CS.

\subsection{Coherent states for associated Hermite and Laguerre polynomials}

The polynomials $\sigma$ and $\tau$ for Hermite and Laguerre polynomials are:

$$
\begin{aligned}
& \sigma(x)=1, \quad \tau(x)=-x, \quad \text { Hermite case } \\
& \sigma(x)=x, \quad \tau(x)=\alpha+1-x, \quad \text { Laguerre case. }
\end{aligned}
$$

In both cases $\sigma^{\prime \prime}=0$ and $\tau^{\prime}=-1$. Then, $r_{m+n-k+1}=-(m+n-k) \sigma^{\prime \prime}-\tau^{\prime}=1$. We have $f\left(r_{m+n-k+1}\right)=f(1)=$ constant $=c$ for any analytical function $f$. The eigenvalues, for any integer $l$, are $\lambda_{l}=-\frac{1}{2} l(l-1) \sigma^{\prime \prime}-l \tau^{\prime}=l$, so that $e_{n}=\lambda_{m+n}-\lambda_{m}=n$. Then

$$
\varepsilon_{n}=e_{1} e_{2} \cdots \mathrm{e}_{n}=1 \cdot 2 \cdot 3 \cdots n=n !
$$

We obtain

$$
\prod_{k=p}^{n+p-1} f\left(r_{m+n-k+1}\right)=c^{n}
$$

The expansion coefficient (37) follows from Eqs (75) and (76) as

$$
K_{n}^{p}(m)=\frac{\Gamma(n+1)}{\sqrt{\Gamma(n+p+1)}} \frac{1}{c^{n}} .
$$

The normalization factor (39) gives here

$$
\mathcal{N}_{p}\left(|z|^{2} ; m\right)=\left[\Gamma(p+1){ }_{1} F_{1}\left(1+p ; 1 ;|c z|^{2}\right)\right]^{-1 / 2},
$$

where ${ }_{1} F_{1}$ is the generalized hypergeometric function. In terms of Meijer's G-function, the normalization factor is given by:

$$
\mathcal{N}_{p}\left(|z|^{2} ; m\right)=\left[G_{1,2}^{1,1}\left(-|c z|^{2} \mid \begin{array}{ccc}
-p & ; & \\
0 & ; & 0
\end{array}\right)\right]^{-1 / 2}
$$


The explicit form of the GPAH-CS relative to Hermite and Laguerre polynomials, defined for any finite $|z|$, follows from (36) :

$$
|z ; m\rangle_{p}=\frac{1}{\sqrt{\Gamma(p+1)_{1} F_{1}\left(p+1 ; 1 ;|c z|^{2}\right)}} \sum_{n=0}^{\infty} \sqrt{\Gamma(n+p+1)} \frac{c^{n} z^{n}}{n !}|n+p\rangle .
$$

For $p=0$, we have $\mathcal{N}_{0}\left(|z|^{2} ; m\right)=\exp \left[-\frac{1}{2}|c z|^{2}\right]$. Performing the variable change $c z \longrightarrow z$, we recover, as expected, for $p=0$, the usual bosonic CS [7],

$$
|z ; m\rangle=\exp \left[\frac{-|z|^{2}}{2}\right] \sum_{n=0}^{\infty} \frac{z^{n}}{\sqrt{n !}}|n\rangle .
$$

Hence, the states (80), for $c=1$, can be considered as the GPAH-CS for the harmonic oscillator.

(i) Non-orthogonality

The inner product $\mathcal{P}={ }_{p^{\prime}}\left\langle z^{\prime} ; m \mid z ; m\right\rangle_{p}$ of two different GPACS-AHF $|z ; m\rangle_{p}$ and $\left|z^{\prime} ; m\right\rangle_{p^{\prime}}$ follows from (40) as

$$
\begin{aligned}
& \mathcal{P}=\mathcal{N}_{p^{\prime}}\left(\left|z^{\prime}\right|^{2} ; m\right) \mathcal{N}_{p}\left(|z|^{2} ; m\right)\left(c z^{\prime}\right)^{\star\left(p-p^{\prime}\right)} \frac{\Gamma(p+1)}{\Gamma\left(p-p^{\prime}+1\right)}{ }_{1} F_{1}\left(p+1 ; p-p^{\prime}+1 ;|c|^{2} z^{\prime \star} z\right) \\
& =\mathcal{N}_{p^{\prime}}\left(\left|z^{\prime}\right|^{2} ; m\right) \mathcal{N}_{p}\left(|z|^{2} ; m\right)\left(c z^{\prime}\right)^{\star\left(p-p^{\prime}\right)} G_{1,2}^{1,1}\left(-|c|^{2} z^{\prime \star} z \mid \begin{array}{ccc}
-p & ; & \\
0 & ; & p^{\prime}-p
\end{array}\right)
\end{aligned}
$$

in terms of generalized hypergeometric and Meijer's G functions, respectively.

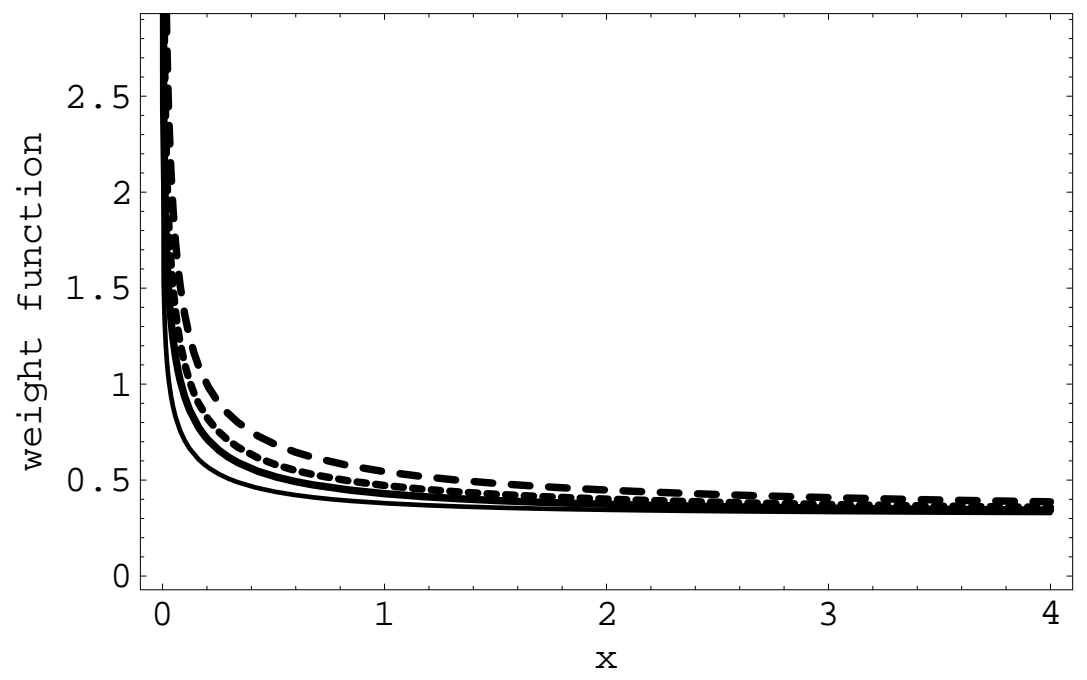

Figure 1. Plots of the weight function (88) of the GPAH-CS (80) versus $x=|z|^{2}$ with $c=1$ and for different values of the photon added number $p$ with $p=1$ (thin solid line), $p=2$ (solid line), $p=3$ (dot line), and $p=4$ (dashed line). 
The relation (48) gives

$$
\int_{0}^{\infty} d x x^{n+p} g_{p}(x ; m)=\left|K_{n}^{p}(m)\right|^{2}=\frac{\Gamma(n+1)^{2}}{\Gamma(n+p+1)} \frac{1}{|c|^{2 n}}
$$

where the function $g_{p}$ is related to the measure $\omega_{p}$ as

$$
\omega_{p}(x ; m)=\frac{x^{p}}{\pi} \frac{1}{\mathcal{N}_{p}^{2}(x ; m)} g_{p}(x ; m) .
$$

Now, performing the variable change $n+m \rightarrow s-1$ transforms the Eq. (84) into:

$$
\int_{0}^{\infty} d x x^{s-1} h_{p}(x ; m)=\frac{1}{|c|^{2 s}} \frac{\Gamma(s-p)^{2}}{\Gamma(s)}
$$

where $h_{p}(x ; m)=g_{p}(x ; m)|c|^{-2(p+1)}$. From the Mellin-inversion theorem (50), we deduce

$$
h_{p}(x ; m)=G_{1,2}^{2,0}\left(|c|^{2} x \mid \begin{array}{lll} 
& ; & 0 \\
-p,-p & ;
\end{array}\right) .
$$

The weight function follows from (79) and (85) as:

$$
\omega_{p}\left(|z|^{2} ; m\right)=\frac{|c|^{2}}{\pi} G_{1,2}^{1,1}\left(-|c z|^{2} \mid \begin{array}{ccc}
-p & ; \\
0 & ; & 0
\end{array}\right) G_{1,2}^{2,0}\left(|c z|^{2} \mid \begin{array}{lll}
0,0 & ;
\end{array}\right)
$$

where we use the multiplication formula of the Meijer's G-function [41]

$$
x^{\alpha} G_{m, n}^{p, q}\left(x \mid \begin{array}{c}
\left(a_{p}\right) \\
\left(b_{q}\right)
\end{array}\right)=G_{m, n}^{p, q}\left(x \mid \begin{array}{c}
\left(a_{p}+\alpha\right) \\
\left(b_{q}+\alpha\right)
\end{array}\right) .
$$

The weight function (88) is positive as confirmed by the Figure 1, where it is represented for $p=1,2,3,4$. The curves show that the measure has a singularity at $x=|z|^{2}=0$ and tends to zero for $x \rightarrow \infty$. For $p=0$, we recover, as expected, the measure $\omega_{0}\left(|z|^{2} ; m\right)=\frac{|c|^{2}}{\pi}$ obtained in our previous work [21] for the corresponding ordinary coherent states.

(iii) Photon number statistics

Taking into account the expressions (77) and (78) of the factors $K_{n}^{p}(m)$ and $\mathcal{N}_{p}\left(|z|^{2} ; m\right)$, we obtain the expectation values of Eqs. (62) and (63):

$$
\begin{aligned}
\left\langle N_{m}\right\rangle & =(m+p) \frac{{ }_{2} F_{2}\left(1+p, m+p+1 ; 1, m+p ;|c z|^{2}\right)}{{ }_{1} F_{1}\left(1+p ; 1 ;|c z|^{2}\right)} \\
\left\langle N_{m}^{2}\right\rangle & =(m+p)^{2} \frac{{ }_{3} F_{3}\left(1+p, m+p+1, m+p+1 ; 1, m+p, m+p ;|c z|^{2}\right)}{{ }_{1} F_{1}\left(1+p ; 1 ;|c z|^{2}\right)} .
\end{aligned}
$$

Then, the Mandel Q-parameter is deduced as:

$$
Q=(m+p)\left[\frac{{ }_{3} \mathcal{F}_{3}\left(|z|^{2} ; m, p\right)}{{ }_{2} \mathcal{F}_{2}\left(|z|^{2} ; m, p\right.}-\frac{{ }_{2} \mathcal{F}_{2}\left(|z|^{2} ; m, p\right)}{{ }_{1} \mathcal{F}_{1}\left(|z|^{2} ; m, p\right)}\right]-1
$$



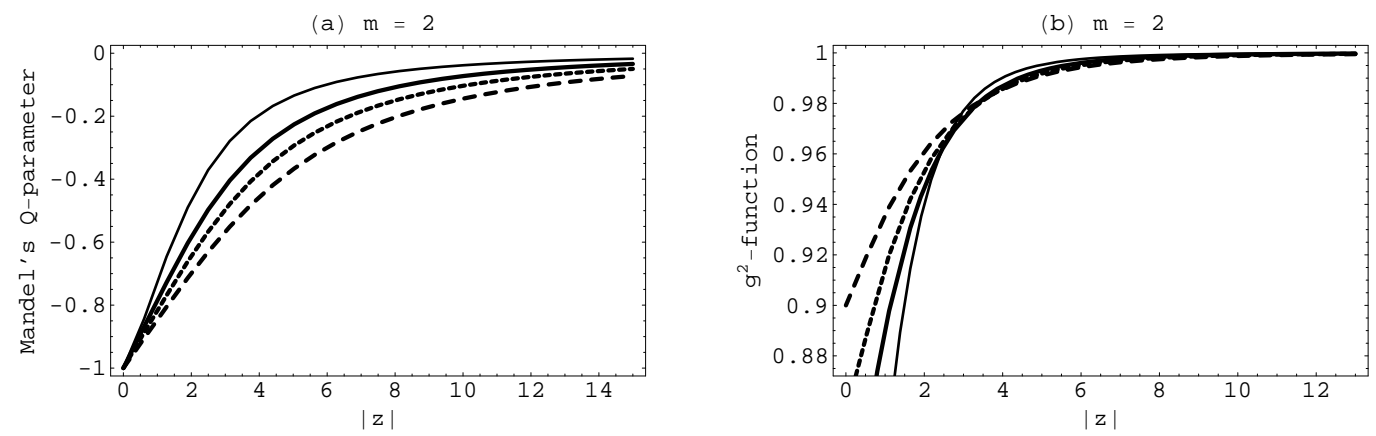

Figure 2. Plots of: (a) the Mandel Q-parameter (92) and (b) the second-order correlation function (93) of the GPAH-CS (80) versus $|z|$ with the derivative order parameter $m=2$ and for various values of the photon-added number $p$ with $p=1$ (thin solid line), $p=3$ (solid line), $p=5$ (dot line) and $p=8$ (dashed line).
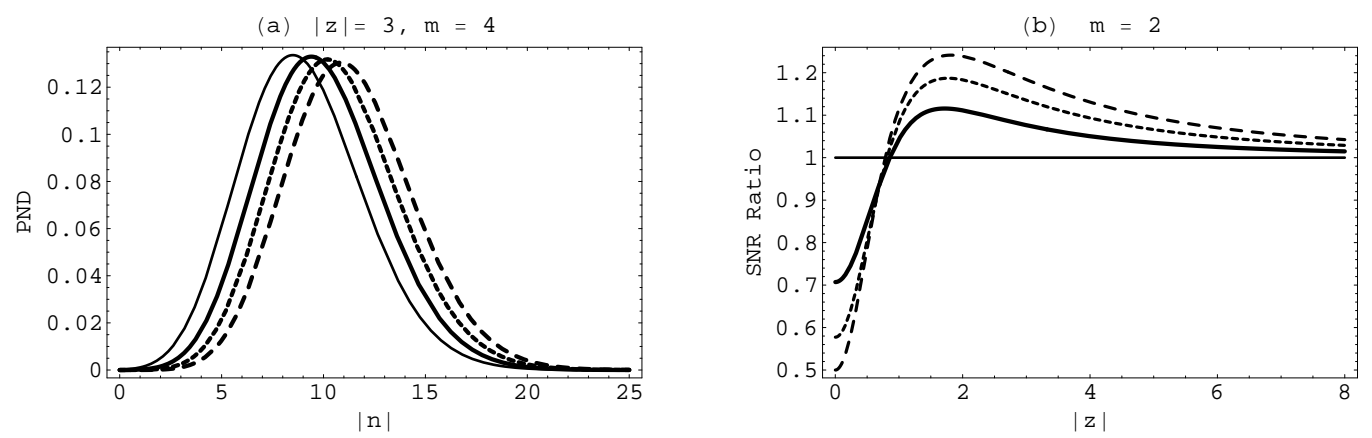

Figure 3. (a) Plot of the PND (94) of the GPAH-CS (80) versus the photon number $n$, for $|z|=3, m=4$ and different values of the photon-added number $p$ with $p=0$ (thin solid line), $p=1$ (solid line), $p=2$ (dot line) and $p=3$ (dashed line).

(b) Plot of the SNR ratios of GPAH-CS (80) to the corresponding GAH-CS versus $|z|$, for various values of the photon-added number $p$ with $p=0$ (thin solid line), $p=1$ (solid line), $p=2$ (dot line) $p=3$ (dashed line).

The second order correlation function (60) follows as

$$
g^{2}={ }_{1} \mathcal{F}_{1}\left(|z|^{2} ; m, p\right) \frac{(m+p){ }_{3} \mathcal{F}_{3}\left(|z|^{2} ; m, p\right)-{ }_{2} \mathcal{F}_{2}\left(|z|^{2} ; m, p\right)}{(m+p){ }_{2} \mathcal{F}_{2}\left(|z|^{2} ; m, p\right)^{2}}
$$

where ${ }_{1} \mathcal{F}_{1},{ }_{2} \mathcal{F}_{2}$ and ${ }_{3} \mathcal{F}_{3}$ are the generalized hypergeometric functions:

$$
\begin{aligned}
& { }_{1} \mathcal{F}_{1}\left(|z|^{2} ; m, p\right)={ }_{1} F_{1}\left(1+p ; 1 ;|c z|^{2}\right) \\
& { }_{2} \mathcal{F}_{2}\left(|z|^{2} ; m, p\right)={ }_{2} F_{2}\left(1+p, m+p+1 ; 1, m+p ;|c z|^{2}\right) \\
& { }_{3} \mathcal{F}_{3}\left(|z|^{2} ; m, p\right)={ }_{3} F_{3}\left(1+p, m+p+1, m+p+1 ; 1, m+p, m+p ;|c z|^{2}\right) .
\end{aligned}
$$

The PND (64) reads as

$$
\mathcal{P}_{n}^{p}\left(|z|^{2} ; m\right)=\frac{\Gamma(n+1)}{\Gamma(p+1){ }_{1} F_{1}\left(1+p ; 1 ;|c z|^{2}\right)} \frac{|c z|^{2(n-p)}}{(n-p) !^{2}}
$$

which gives for $p=0$

$$
\mathcal{P}_{n}^{p}\left(|z|^{2} ; m\right)=e^{-|c z|^{2}} \frac{|c z|^{2 n}}{n !}
$$


Finally the SNR (65) gives

$\sigma^{p}\left(|z|^{2} ; m\right)=(m+p) \frac{{ }_{2} \mathcal{F}_{2}\left(|z|^{2} ; m, p\right)-p_{1} F_{1}\left(1+p ; 1 ;|c z|^{2}\right)}{(m+p) \sqrt{{ }_{3} \mathcal{F}_{3}\left(|z|^{2} ; m, p\right)_{1} \mathcal{F}_{1}\left(|z|^{2} ; m, p\right)-\left({ }_{2} \mathcal{F}_{2}\left(|z|^{2} ; m, p\right)\right)^{2}}}$.

(iv) Thermal statistics

The relative normalized density operator and the partition function are provided as

$$
\begin{aligned}
\rho^{(p)} & =\frac{1}{Z} \sum_{n=0} e^{-\beta n}|n+p\rangle\langle n+p| \\
& =\frac{1}{\bar{n}_{o}+1} \sum_{n=0}\left(\frac{\bar{n}_{o}}{\bar{n}_{o}+1}\right)^{n}|n+p\rangle\langle n+p|, \quad Z=\frac{1}{1-e^{-\beta}}:=\bar{n}_{o}+1
\end{aligned}
$$

respectively, with $\bar{n}_{o}=\left(e^{\beta}-1\right)^{-1}$ corresponding to the thermal expectation value of the number operator, i.e. the Bose-Einstein distribution function for oscillators with angular frequency $\omega=1$, where $\hbar=1$.

From (80) and (98), we get, in terms of generalized hypergeometric functions, the $Q$-distribution

$$
{ }_{p}\left\langle z ; m\left|\rho^{(p)}\right| z ; m\right\rangle=\left(\bar{n}_{o}+1\right) \frac{{ }_{1} F_{1}\left(p+1 ; 1 ;|c z|^{2} e^{-\beta}\right)}{{ }_{1} F_{1}\left(p+1 ; 1 ;|c z|^{2}\right)}
$$

and in terms of Meijer's G functions,

$$
{ }_{p}\left\langle z ; m\left|\rho^{(p)}\right| z ; m\right\rangle_{p}=\left(\bar{n}_{o}+1\right) \frac{G_{1,2}^{1,1}\left(-|c z|^{2} e^{-\beta} \mid \begin{array}{ccc}
-p & ; & \\
0 & ; & 0
\end{array}\right)}{G_{1,2}^{1,1}\left(-|c z|^{2} \mid \begin{array}{ccc}
-p & ; & \\
0 & ; & 0
\end{array}\right)} .
$$

After performing the angular integration and taking $x=|c z|^{2}$, the condition (68) leads to

$$
\operatorname{Tr} \rho^{(p)}=\frac{1}{Z} \int_{0}^{\infty} d x x^{p} G_{1,2}^{1,1}\left(x \mid \begin{array}{cc|c}
-p & ; & \\
0 & ; & 0
\end{array}\right) G_{1,2}^{1,1}\left(-x e^{-\beta} \mid \begin{array}{ccc}
-p & ; & \\
0 & ; & 0
\end{array}\right) .
$$

Thus, using the properties of the integral of Meijer's G-functions products provides the partition function expression

$$
Z=\frac{1}{1-e^{-\beta}}=\bar{n}_{o}+1 \text {. }
$$

From (69), using the result

$$
\left\langle n+p\left|\rho^{(p)}\right| n+p\right\rangle=\frac{1}{\bar{n}_{o}+1}\left(\frac{\bar{n}_{o}}{\bar{n}_{o}+1}\right)^{n}
$$

we obtain the following integration equality

$$
\frac{1}{\bar{n}_{o}+1}\left(\frac{\bar{n}_{o}}{\bar{n}_{o}+1}\right)^{n} \frac{1}{|c|^{2(n+p+1)}} \frac{\Gamma(n+1)^{2}}{\Gamma(n+p+1)}=\int_{0}^{\infty} d x x^{n+p} P(x) G_{1,2}^{2,0}\left(\left.|c|^{2} x\right|_{-p,-p} \quad ; \quad 0\right) \text {. }
$$

After performing the exponent change $n+p=s-1$ of $x=|z|^{2}$, in order to get to the Stieltjes moment problem, we arrive at the $P$-function obtained as

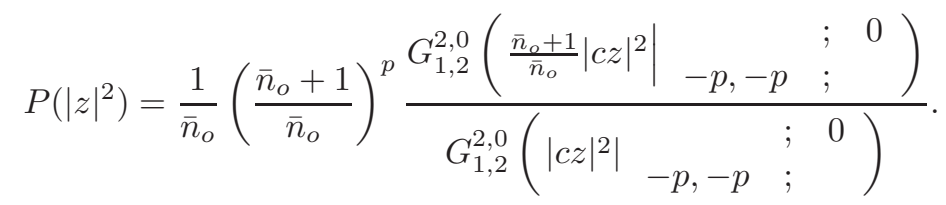


The resulting $P$-function (104) obeys the normalized to unity condition (70). Thereby, the diagonal representation of the normalized density operator in terms of the GPAH-CS projector (69), in this case, is

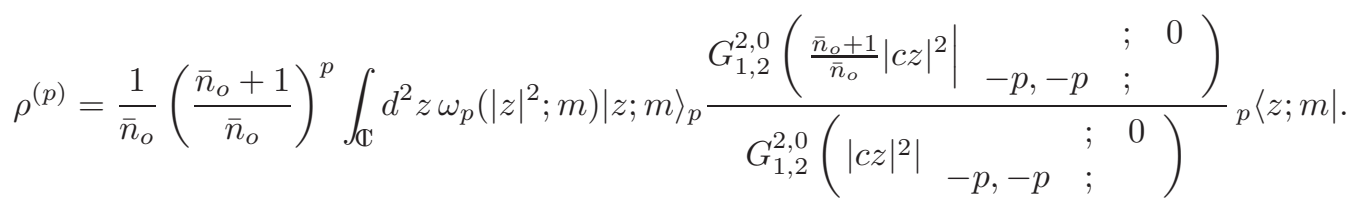

With the relations (90), (91) and the definition (71), the pseudo-thermal expectation values of the number operator and of its square are obtained as

$$
\begin{aligned}
& \left\langle N_{m}\right\rangle^{(p)}=\operatorname{Tr}\left(\rho^{(p)} N_{m}\right)=\frac{1}{\bar{n}_{o}+1}(m+p) \sum_{n=0}^{\infty}\left(1+\frac{n}{m+p+1}\right)\left(\frac{\bar{n}_{o}}{\bar{n}_{o}+1}\right)^{n} \\
& \left\langle N_{m}^{2}\right\rangle^{(p)}=\operatorname{Tr}\left(\rho^{(p)} N_{m}^{2}\right)=\frac{1}{\bar{n}_{o}+1}(m+p)^{2} \sum_{n=0}^{\infty}\left(1+\frac{n}{m+p+1}\right)^{2}\left(\frac{\bar{n}_{o}}{\bar{n}_{o}+1}\right)^{n} .
\end{aligned}
$$

Using (106), the thermal intensity correlation function (72), is obtained as

$$
\left(g^{2}\right)^{(p)}=1-\frac{\left[(m+p+1)^{2}+\bar{n}_{o}\right]-\bar{n}_{o}^{2}(m+p)}{(m+p)\left[(m+p+1)+\bar{n}_{o}\right]^{2}} .
$$

Thereby, the thermal analogue of the Mandel parameter (73) is

$$
Q^{(p)}=\frac{\bar{n}_{o}^{2}(m+p)-\left[(m+p+1)^{2}+\bar{n}_{o}\right]}{(m+p+1)\left[(m+p+1)+\bar{n}_{o}\right]} .
$$

\subsection{Coherent states for associated Jacobi polynomials and hypergeometric functions}

The polynomial coefficients $\sigma$ and $\tau$ ) for Jacobi and hypergeometric cases are:

$$
\begin{array}{lll}
\sigma(x)=1-x^{2}, & \tau(x)=(\beta-\alpha)-(\alpha+\beta+2) x, & \text { for Jacobi polynomials } \\
\sigma(x)=x(1-x), & \tau(x)=(\alpha+1)-(\alpha+\beta+2) x, & \text { for Hypergeometric functions }
\end{array}
$$

In both the cases, $\sigma^{\prime \prime}=-2$ and $\tau^{\prime}=-(\alpha+\beta+2)=-\mu$. The eigenvalues, for any integer $l$, are

$$
\lambda_{l}=-\frac{1}{2} l(l-1) \sigma^{\prime \prime}-l \tau^{\prime}=l(l+\mu-1), \quad e_{n}=\lambda_{m+n}-\lambda_{m}=n(n+2 m+\mu-1) .
$$

Then the quantities $\varepsilon_{n}$, for any integer $n$, can be read as:

$$
\varepsilon_{n}=e_{1} e_{2} \cdots \mathrm{e}_{n}=\Gamma(n+1) \frac{\Gamma(n+2 m+\mu)}{\Gamma(2 m+\mu)} .
$$

The variable $r_{m+n-k+1}$ of the functional $f$ in the expansion coefficient (37) is written as:

$$
r_{m+n-k+1}=-(m+n-k) \sigma^{\prime \prime}-\tau^{\prime}=2(m+n-k)+\mu
$$

The explicit form of the GPAH-CS depends on the expansion coefficient $K_{n}^{p}(m)$ which is defined in terms of the functional $f$. In the following, we adopt some functionals close to those used in [20]. 


\subsubsection{Case where $f$ is constant}

First, we consider that the analytical function $f$ is a constant $c$. Then, we have $f\left(r_{m+n-k+1}\right)=$ constant $=c$, and

$$
\prod_{k=p}^{n+p-1} f\left(r_{m+n-k+1}\right)=c^{n}
$$

The expansion coefficient (37) from Eqs (111) and (113) becomes

$$
K_{n}^{p}(m)=\frac{\Gamma(n+1) \Gamma(n+2 m+\mu)}{\sqrt{\Gamma(2 m+\mu) \Gamma(n+p+1) \Gamma(n+p+2 m+\mu)}} \frac{1}{c^{n}} .
$$

Without loss of generality in the sequel, we take $c=1$. Then, the expansion coefficient gives

$$
K_{n}^{p}(m)=\frac{\Gamma(n+1) \Gamma(n+2 m+\mu)}{\sqrt{\Gamma(2 m+\mu) \Gamma(n+p+1) \Gamma(n+p+2 m+\mu)}} .
$$

For $p=0$, this reduces to

$$
K_{n}^{0}(m)=\sqrt{\frac{\Gamma(n+1) \Gamma(n+2 m+\mu)}{\Gamma(2 m+\mu)}},
$$

and we recover the result of the corresponding CS, obtained in [21].

The normalization factor (39) is formulated in terms of the generalized hypergeometric function ${ }_{2} F_{3}$

$\mathcal{N}_{p}\left(|z|^{2} ; m\right)=\left[\frac{\Gamma(p+1) \Gamma(2 m+p+\mu)}{\Gamma(2 m+\mu)}{ }_{2} F_{3}\left(1+p, 2 m+p+\mu ; 1,2 m+\mu, 2 m+\mu ;|z|^{2}\right)\right]^{-1 / 2}$,

or in terms of Meijer G-function

$\mathcal{N}_{p}\left(|z|^{2} ; m\right)=\left[\Gamma(2 m+\mu) G_{2,4}^{1,2}\left(\begin{array}{l|l}-|z|^{2} & \begin{array}{l}-p, 1-2 m-p-\mu \\ 0,0,1-2 m-\mu, 1-2 m-\mu\end{array}\end{array}\right]^{-1 / 2}\right.$.

where from now and in the sequel we use the more compact notation of long Meijer's G-function.

$$
G_{p, q}^{m, n}\left(x \mid \begin{array}{c}
a_{1}, \ldots, a_{p} \\
b_{1}, \ldots, b_{q}
\end{array}\right)
$$

For $p=0$, it is reduced to the result obtained in [21] for the corresponding CS, expressed with

$\left.\mathcal{N}_{0}\left(|z|^{2} ; m\right)={ }_{0} F_{1}\left(2 m+\mu,|z|^{2}\right)\right]^{-1 / 2}=\left[|z|^{1-\mu-2 m} I_{2 m+\mu-1}(2|z|) \Gamma(2 m+\mu)\right]^{-1 / 2}$.

${ }_{0} F_{1}$ is the confluent hypergeometric function and $I_{\nu^{-}}$the modified Bessel function of the first kind.

The explicit form of the GPAH-CS relative to Jacobi and Hypergeometric polynomials, defined for any finite $|z|$, follows from (36):

$$
|z ; m\rangle_{p}=\mathcal{N}_{p}\left(|z|^{2} ; m\right) \sum_{n=0}^{\infty} \sqrt{\frac{\Gamma(2 m+\mu) \Gamma(n+p+1) \Gamma(n+p+2 m+\mu)}{\Gamma(n+2 m+\mu)^{2}}} \frac{z^{n}}{n !}|n+p\rangle .
$$


For $p=0$, we recover the corresponding CS obtained in [21],

$$
|z ; m\rangle=\frac{1}{\sqrt{{ }_{0} F_{1}\left(2 m+\mu,|z|^{2}\right)}} \sum_{n=0}^{\infty} \frac{z^{n}}{\sqrt{\Gamma(n+1)(2 m+\mu)_{n}}}|n\rangle, \quad|z|<\infty
$$

as a particular case.

(i) Non-orthogonality

The inner product $\mathcal{P}={ }_{p^{\prime}}\left\langle z^{\prime} ; m \mid z ; m\right\rangle_{p}$ of two different GPAH-CS $|z ; m\rangle_{p}$ and $\left|z^{\prime} ; m\right\rangle_{p^{\prime}}$, for Jacobi polynomials and hypergeometric functions, follows from (40) as

$$
\begin{aligned}
\mathcal{P}= & \mathcal{N}_{p^{\prime}}\left(\left|z^{\prime}\right|^{2} ; m\right) \mathcal{N}_{p}\left(|z|^{2} ; m\right) z^{\prime \star\left(p-p^{\prime}\right)} \frac{\Gamma(p+1) \Gamma(p+2 m+\mu)}{\Gamma\left(p-p^{\prime}+1\right) \Gamma\left(p-p^{\prime}+2 m+\mu\right)} \times \\
& { }_{2} F_{3}\left(p+1,2 m+p+\mu ; p-p^{\prime}+1,2 m+\mu, 2 m+\mu+p-p^{\prime} ; z^{\prime \star} z\right)
\end{aligned}
$$

yielding, for $p=p^{\prime}=0$,

$$
{ }_{0}\left\langle z^{\prime} ; m \mid z ; m\right\rangle_{0}=\frac{{ }_{0} F_{1}\left(2 m+\mu, z^{\prime \star} z\right)}{\sqrt{{ }_{0} F_{1}\left(2 m+\mu,\left|z^{\prime}\right|^{2}\right)_{0} F_{1}\left(2 m+\mu,|z|^{2}\right)}} .
$$

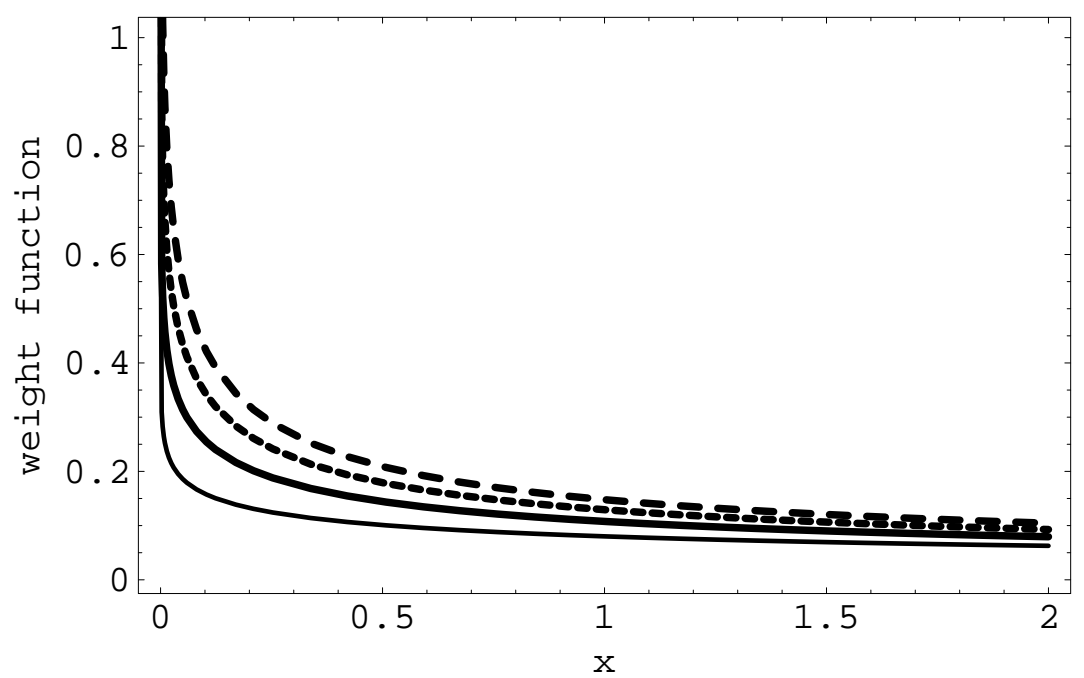

Figure 4. Plots of the weight function (128) of the GPAH-CS (121) versus $x=|z|^{2}$ with parameters $m=4, \mu=1.5$ and for different values of the photon added number $p$ with $p=1$ (thin solid line), $p=2$ (solid line), $p=3$ (dot line), and $p=4$ (dashed line).

(ii) Overcompleteness

The relation (48) now engenders for the Jacobi polynomials and hypergeometric functions:

$$
\int_{0}^{\infty} d x x^{n+p} g_{p}(x ; m)=\frac{\Gamma(n+1)^{2} \Gamma(n+2 m+\mu)^{2}}{\Gamma(2 m+\mu) \Gamma(n+p+1) \Gamma(n+p+2 m+\mu)}
$$


where the function $g_{p}$ is related to the measure $\omega_{p}$ by (85). Provided the variable change $n+m \rightarrow s-1$, it becomes:

$$
\int_{0}^{\infty} d x x^{s-1} h_{p}(x ; m)=\frac{\Gamma(s-p)^{2} \Gamma(s-p-1+2 m+\mu)^{2}}{\Gamma(s) \Gamma(s-1+2 m+\mu)}
$$

where $h_{p}(x ; m)=g_{p}(x ; m) \Gamma(2 m+\mu)$. From the Mellin-inversion theorem (50), we deduce

$h_{p}(x ; m)=G_{2,4}^{4,0}\left(\begin{array}{l|l}x & \begin{array}{l}0,-1+2 m+\mu \\ -p,-p,-1-p+2 m+\mu,-1-p+2 m+\mu\end{array}\end{array}\right)$.

The weight function follows from (85) and (118) as

$$
\begin{aligned}
\omega_{p}\left(|z|^{2} ; m\right)= & \frac{1}{\pi} G_{2,4}^{1,2}\left(-|z|^{2} \mid \begin{array}{l}
-p, 1-2 m-p-\mu \\
0,0,1-2 m-\mu, 1-2 m-\mu
\end{array}\right) \times \\
& \times G_{2,4}^{4,0}\left(|z|^{2} \mid \begin{array}{l}
p,-1+p+2 m+\mu \\
0,0,-1+2 m+\mu,-1+2 m+\mu
\end{array}\right)
\end{aligned}
$$

using the multiplication formula of the Meijer's G-function (89)). The weight function (128) is positive for the parameter $\mu>0$ as shown on the representations in Figure 4 with $\mu=1.5$ and for different values of the photon-added number $p=1,2,3,4$. Figure 4 also shows that the weight function (128) presents a singularity at $x=0$ and tends to zero for $x \rightarrow \infty$. For $p=0$, the Meijer $\mathrm{G}$ function $G_{2,4}^{4,0}$ reduces to $2|z|^{-1+\mu+2 m} K_{2 m+\mu-1}(2|z|)$, and taking in to account (120), we retrieve the measure obtained in [21] for the corresponding ordinary coherent states,

$$
\omega_{0}\left(|z|^{2} ; m\right)=\frac{2}{\pi} I_{2 m+\mu-1}(2|z|) K_{2 m+\mu-1}(2|z|),
$$

where $I_{\nu}$ and $K_{\nu}$ are the modified Bessel functions of first and second kind, respectively.

(iii) Photon number statistics
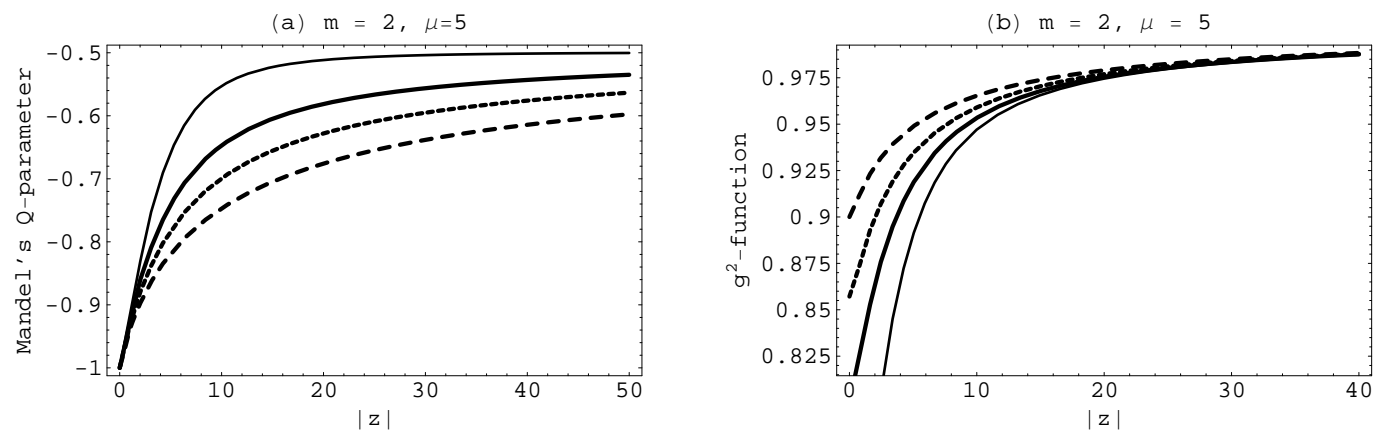

Figure 5. Plots of: (a) the Mandel Q-parameter (132) and (b) the second order correlation function (133) of the GPAH-CS (121) versus $|z|$ with parameters $m=2, \mu=5$ and for various values of the photon-added number $p$ with $p=1$ (thin solid line), $p=3$ (solid line), $p=5$ (dot line) and $p=8$ (dashed line). 

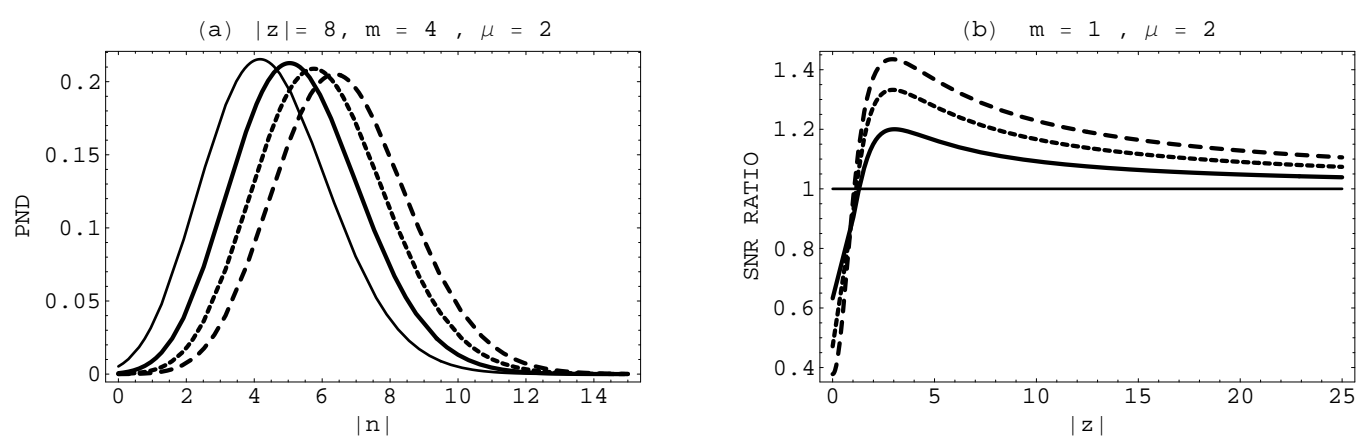

Figure 6. (a) Plot of the PND (134) of the GPAH-CS (121) versus the photon number $n$, with the parameters $m=4, \mu=2$ and for different values of the photon-added number $p$ with $p=0$ (thin solid line), $p=1$ (solid line), $p=2$ (dot line) and $p=3$ (dashed line).

(b) Plot of the SNR (136) ratios of GPAH-CS (121) and the corresponding GAH-CS versus $|z|$, with the parameters $m=1, \mu=2$, for various values of the photon-added number $p$ with $p=0$ (thin solid line), $p=1$ (solid line), $p=2$ (dot line) and $p=3$ (dashed line)

Taking into account the expressions (115) and (117) of the factors $K_{n}^{p}(m)$ and $\mathcal{N}_{p}\left(|z|^{2} ; m\right)$, we obtain the expectation values $(62,63)$ :

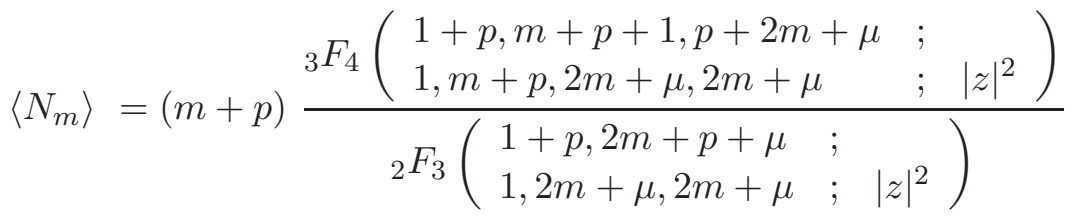

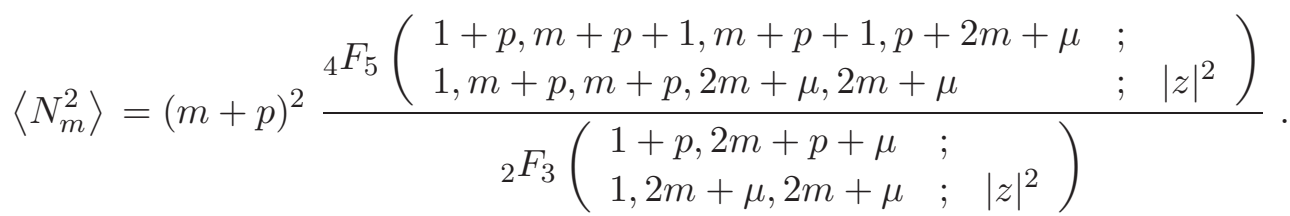

where we adopt from now and in the sequel the following notation

$$
{ }_{p} F_{q}\left(\begin{array}{cc}
a_{1}, \ldots, a_{p} & ; \\
b_{1}, \ldots, b_{q} & ; x
\end{array}\right) .
$$

for long ${ }_{p} F_{q}$ hypergeometric. Then, the Mandel Q-parameter can be inferred as:

$$
Q=(m+p)\left[\frac{{ }_{4} \mathcal{F}_{5}\left(|z|^{2} ; m, p\right)}{{ }_{3} \mathcal{F}_{4}\left(|z|^{2} ; m, p\right.}-\frac{{ }_{3} \mathcal{F}_{4}\left(|z|^{2} ; m, p\right)}{{ }_{2} \mathcal{F}_{3}\left(|z|^{2} ; m, p\right)}\right]-1
$$

while the second order correlation function (60) yields

$$
g^{2}={ }_{2} \mathcal{F}_{3}\left(|z|^{2} ; m, p\right) \frac{(m+p){ }_{4} \mathcal{F}_{5}\left(|z|^{2} ; m, p\right)-{ }_{3} \mathcal{F}_{4}\left(|z|^{2} ; m, p\right)}{(m+p){ }_{3} \mathcal{F}_{4}\left(|z|^{2} ; m, p\right)^{2}}
$$

where ${ }_{2} \mathcal{F}_{3},{ }_{3} \mathcal{F}_{4}$ and ${ }_{4} \mathcal{F}_{5}$ are the generalized hypergeometric functions:

$$
\begin{aligned}
& { }_{2} \mathcal{F}_{3}\left(|z|^{2} ; m, p\right)={ }_{2} F_{3}\left(\begin{array}{ll}
1+p, 2 m+p+\mu & ; \\
1,2 m+\mu, 2 m+\mu & ; \quad|z|^{2}
\end{array}\right) \\
& { }_{3} \mathcal{F}_{4}\left(|z|^{2} ; m, p\right)={ }_{3} F_{4}\left(\begin{array}{ll}
1+p, m+p+1, p+2 m+\mu & ; \\
1, m+p, 2 m+\mu, 2 m+\mu & ; \quad|z|^{2}
\end{array}\right)
\end{aligned}
$$




$$
{ }_{4} \mathcal{F}_{5}\left(|z|^{2} ; m, p\right)={ }_{4} F_{5}\left(\begin{array}{ll}
1+p, m+p+1, m+p+1, p+2 m+\mu & ; \\
1, m+p, m+p, 2 m+\mu, 2 m+\mu & ; \quad|z|^{2}
\end{array}\right) .
$$

The PND (64) reads as

$$
\mathcal{P}_{n}^{p}\left(|z|^{2} ; m\right)=\frac{\Gamma(2 m+\mu)^{2} \Gamma(n+2 m+\mu) \Gamma(n+1)}{\Gamma(p+2 m+\mu) \Gamma(p+1) \Gamma(n-p+2 m+\mu)^{2}{ }_{2} \mathcal{F}_{3}\left(|z|^{2} ; m, p\right)} \frac{|z|^{2(n-p)}}{\left(n_{p}\right) !^{2}} .
$$

This gives, for $p=0$, the result

$\mathcal{P}_{n}^{p}\left(|z|^{2} ; m\right)=\frac{\Gamma(2 m+\mu)}{\Gamma(n+2 m+\mu)_{0} F_{1}\left(2 m+\mu,|z|^{2}\right)} \frac{|z|^{2 n}}{n !}$

corresponding to the conventional GAH-CS PND. Finally the SNR (65) gives

$\sigma^{p}\left(|z|^{2} ; m\right)=\frac{(m+p){ }_{3} \mathcal{F}_{4}\left(|z|^{2} ; m, p\right)-p_{2} F_{3}\left(1+p ; 1 ;|z|^{2}\right)}{(m+p) \sqrt{{ }_{4} \mathcal{F}_{5}\left(|z|^{2} ; m, p\right)_{2} \mathcal{F}_{3}\left(|z|^{2} ; m, p\right)-\left({ }_{3} \mathcal{F}_{4}\left(|z|^{2} ; m, p\right)\right)^{2}}}$.

(iv) Thermal statistics

Consider the normalized density operator expression

$$
\rho^{(p)}=\frac{1}{Z} \sum_{n=0} e^{-\beta e_{n}}|n+p\rangle\langle n+p|
$$

in which the exponent $\beta e_{n}$ is recast as follows

$$
\beta e_{n}=\beta\left[n^{2}+(2 m+\mu-1) n\right]=A n^{2}-B_{m, \mu} n
$$

where $A=\beta, B_{m, \mu}=-\beta(2 m+\mu-1)$. Then, the energy exponential can be expanded in the power series, (see for e.g., [28]) such that

$$
\begin{aligned}
e^{-\beta e_{n}}=e^{-A n}\left[\sum_{k=0}^{\infty} \frac{\left(B_{m, \mu}\right)^{k}}{k !} n^{2 k}\right] & =\left\{\sum_{k=0}^{\infty} \frac{\left(B_{m, \mu}\right)^{k}}{k !}\left(\frac{d}{d A}\right)^{2 k}\right\}\left(e^{-A}\right)^{n} \\
& =\exp \left[B_{m, \mu}\left(\frac{d}{d A}\right)^{2}\right]\left(e^{-A}\right)^{n} .
\end{aligned}
$$

Thereby,

$$
\rho^{(p)}=\frac{\exp \left[B_{m, \mu}\left(\frac{d}{d A}\right)^{2}\right]}{Z} \sum_{n=0}^{\infty}\left(e^{-A}\right)^{n}|n+p\rangle\langle n+p| .
$$

From (121) and (140), we get, in terms of generalized hypergeometric functions, the $Q$-distribution or Husimi distribution

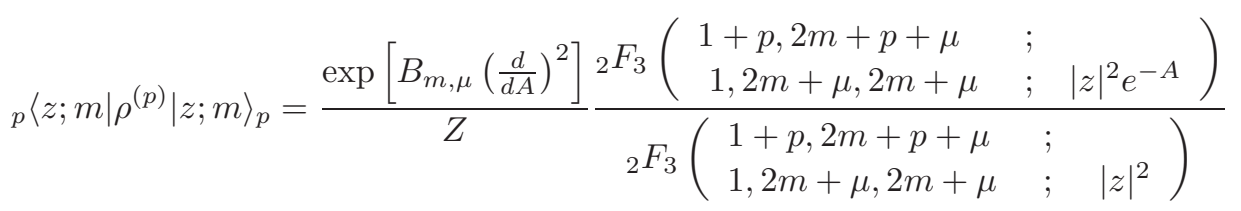

and in terms of Meijer's G functions,

${ }_{p}\left\langle z ; m\left|\rho^{(p)}\right| z ; m\right\rangle_{p}=\frac{\exp \left[B_{m, \mu}\left(\frac{d}{d A}\right)^{2}\right]}{Z} \frac{G_{2,4}^{1,2}\left(-|z|^{2} e^{-A} \mid \begin{array}{l}-p, 1-2 m-p-\mu \\ 0,0,1-2 m-\mu, 1-2 m-\mu\end{array}\right)}{G_{2,4}^{1,2}\left(-|z|^{2} \mid \begin{array}{l}-p, 1-2 m-p-\mu \\ 0,0,1-2 m-\mu, 1-2 m-\mu\end{array}\right)}$. 
The angular integration achieved, taking $x=|z|^{2}$, the condition (68) supplies

$$
\begin{aligned}
\operatorname{Tr} \rho^{(p)} & =\frac{\exp \left[B_{m, \mu}\left(\frac{d}{d A}\right)^{2}\right]}{Z} \int_{0}^{\infty} d x x^{p} G_{2,4}^{1,2}\left(\begin{array}{l|l}
-x e^{-A} & \begin{array}{l}
-p, 1-2 m-p-\mu \\
0,0,1-2 m-\mu, 1-2 m-\mu
\end{array}
\end{array}\right) \times \\
& \times G_{2,4}^{4,0}\left(x \mid \begin{array}{l}
0,-1+2 m+\mu \\
-p,-p,-1-p+2 m+\mu,-1-p+2 m+\mu
\end{array}\right) .
\end{aligned}
$$

Then, the integral of Meijer's G-functions products properties provides the partition function expression

$$
Z=\exp \left[B_{m, \mu}\left(\frac{d}{d A}\right)^{2}\right] \sum_{n=0}^{\infty}\left(e^{-A}\right)^{n} .
$$

From (69), using the result

$$
\left\langle n+p\left|\rho^{(p)}\right| n+p\right\rangle=\frac{\exp \left[B_{m, \mu}\left(\frac{d}{d A}\right)^{2}\right]\left(e^{-A}\right)^{n}}{Z}
$$

and setting $\bar{n}_{A}=\left(e^{A}-1\right)^{-1}$, we get the following integration equality

$$
\begin{aligned}
& \frac{1}{\bar{n}_{A}+1}\left(\frac{\bar{n}_{A}}{\bar{n}_{A}+1}\right)^{n} \frac{\Gamma(n+1)^{2} \Gamma(n+2 m+\mu)^{2}}{\Gamma(n+p+1) \Gamma(n+p+2 m+\mu)}=\int_{0}^{\infty} d x x^{n+p} P(x) \times \\
& G_{2,4}^{4,0}\left(x \mid \begin{array}{l}
0,-1+2 m+\mu \\
-p,-p,-1-p+2 m+\mu,-1-p+2 m+\mu
\end{array}\right) .
\end{aligned}
$$

After performing the exponent change $n+p=s-1$ of $x=|z|^{2}$, in order to get the Stieltjes moment problem, we arrive at the $P$-function obtained as

$$
P\left(|z|^{2}\right)=\frac{1}{\bar{n}_{A}}\left(\frac{\bar{n}_{A}+1}{\bar{n}_{A}}\right)^{p} \frac{G_{2,4}^{4,0}\left(\frac{\bar{n}_{A}+1}{\bar{n}_{A}}|z|^{2} \mid \begin{array}{l}
0,-1+2 m+\mu \\
-p,-p,-1-p+2 m+\mu,-1-p+2 m+\mu
\end{array}\right)}{G_{2,4}^{4,0}\left(|z|^{2} \mid \begin{array}{l}
0,-1+2 m+\mu \\
-p,-p,-1-p+2 m+\mu,-1-p+2 m+\mu
\end{array}\right)}
$$

which obeys the normalization to unity condition (70) .

Then, the diagonal representation of the normalized density operator in terms of the GPAH-CS projector (69) takes the form

$\rho^{(p)}=\frac{1}{\bar{n}_{A}}\left(\frac{\bar{n}_{A}+1}{\bar{n}_{A}}\right)^{p} \int_{\mathbb{C}} d^{2} z \omega_{p}\left(|z|^{2} ; m\right)|z ; m\rangle_{p} \mathfrak{S}_{2,4}^{4,0}\left(|z|^{2} ; \bar{n}_{A}\right)_{p}\langle z ; m|$

with $\mathfrak{S}_{2,4}^{4,0}\left(|z|^{2}, \bar{n}_{A}\right)$ the Meijer's G-functions quotient given in (145). Using the relations (129), (130), and the definition (71), the pseudo-thermal expectation values of the number operator and of its square coincide with (106), where $\bar{n}_{o}$ is replaced by $\bar{n}_{A}$. Therefore, the thermal intensity correlation function (72) and the thermal analogue of the Mandel parameter (73) have identical expressions as in (107) and (108), respectively, with $\bar{n}_{A}$ instead of $\bar{n}_{o}$.

\subsubsection{Case where $f$ is not constant}

Consider the linear function $\xi$ depending on the variable $r_{m, n}(k) \equiv r_{m+n-k+1}$ :

$$
\xi\left(r_{m, n}(k) ; b ; d\right)=b r_{m, n}(k)+d=2 b(m+n+\nu-k)+d
$$


where we set $\mu=2 \nu$, and the the product:

$$
\prod_{k=0}^{n-1} \xi\left(r_{m, n}(k) ; b ; d\right)=\prod_{k=0}^{n-1}(c(m+n+\nu-k+d / c))
$$

where we put $c=2 b$. After a straightforward computation, we obtain:

$$
\prod_{k=0}^{n-1} \xi\left(r_{m, n}(k) ; c ; d\right)=(-c)^{n}(-m-n-\nu-d / c)_{n} .
$$

Using the result $(-a)_{k}=(-1)^{k} \frac{a !}{(a-k) !}$, we have:

$$
\prod_{k=0}^{n-1} \xi\left(r_{m, n}(k) ; c ; d\right)=(-c)^{n} \frac{\Gamma(m+n+\nu+d / c+1)}{\Gamma(m+\nu+d / c+1)} .
$$

We consider as example of non constant function : $f\left(r_{m, n}(k)\right)=\sqrt{\xi\left(r_{m, n}(k) ;-1,1\right)}$. We find

$$
\prod_{k=0}^{n-1} f\left(r_{m, n}(k)=\sqrt{\frac{\Gamma(n+m+\nu)}{\Gamma(m+\nu)}},\right.
$$

and the expansion coefficient reads:

$$
K_{n}^{p}(m)=\sqrt{\frac{\Gamma(n+1)^{2} \Gamma(n+2 m+2 \nu)^{2} \Gamma(m+\nu)}{\Gamma(2 m+2 \nu) \Gamma(n+p+1) \Gamma(n+p+2 m+2 \nu) \Gamma(n+m+\nu)}} .
$$

(i) Normalization

The normalization factor (39) becomes:

$\mathcal{N}_{p}\left(|z|^{2} ; m\right)=\left[\frac{\Gamma(p+1) \Gamma(2 m+p+2 \nu)}{\Gamma(2 m+2 \nu)}{ }_{3} F_{3}\left(\begin{array}{ll}1+p, 2 m+p+2 \nu, m+\nu & ; \\ 1,2 m+2 \nu, 2 m+2 \nu & ; \quad|z|^{2}\end{array}\right)\right]^{-1 / 2}$,

where ${ }_{3} F_{3}$ is the generalized hypergeometric function. In terms of Meijer's G-function

$\mathcal{N}_{p}\left(|z|^{2} ; m\right)=\left[\begin{array}{l|l}\Gamma(2 m+2 \nu) G_{3,4}^{1,3}\end{array}\left(-|z|^{2} \mid \begin{array}{l}-p, 1-2 m-p-2 \nu, 1-m-\nu \\ 0,0,1-2 m-2 \nu, 1-2 m-\nu\end{array}\right)\right]^{-1 / 2}$

For $p=0$,

$$
\begin{aligned}
\mathcal{N}_{0}\left(|z|^{2} ; m\right) & =\left[{ }_{1} F_{1}\left(m+\nu ; m+\nu ;|z|^{2}\right)\right]^{-1 / 2} \\
& =\left[2^{2 m+2 \nu-1}|z|^{\frac{1}{2}-\nu-m} e^{\frac{1}{2}|z|^{2}} I_{m+\nu-1 / 2}\left(\frac{1}{2}|z|^{2}\right) \Gamma\left(m+\nu+\frac{1}{2}\right)\right]^{-1 / 2},
\end{aligned}
$$

where ${ }_{1} F_{1}$ is the confluent hypergeometric function, $I_{\nu}$-the modified Bessel function of the first kind.

The explicit form of the GPAH-CS corresponding to the Jacobi polynomials and hypergeometric functions, defined for any finite $|z|$, follows from (36):

$$
\begin{aligned}
|z ; m\rangle_{p}= & \mathcal{N}_{p}\left(|z|^{2} ; m\right) \times \\
& \times \sum_{n=0}^{\infty} \sqrt{\frac{\Gamma(2 m+2 \nu) \Gamma(n+p+1) \Gamma(n+p+2 m+2 \nu) \Gamma(n+m+\nu)}{\Gamma(n+2 m+2 \nu)^{2} \Gamma(m+\nu)}} \frac{z^{n}}{n !}|n+p\rangle .
\end{aligned}
$$




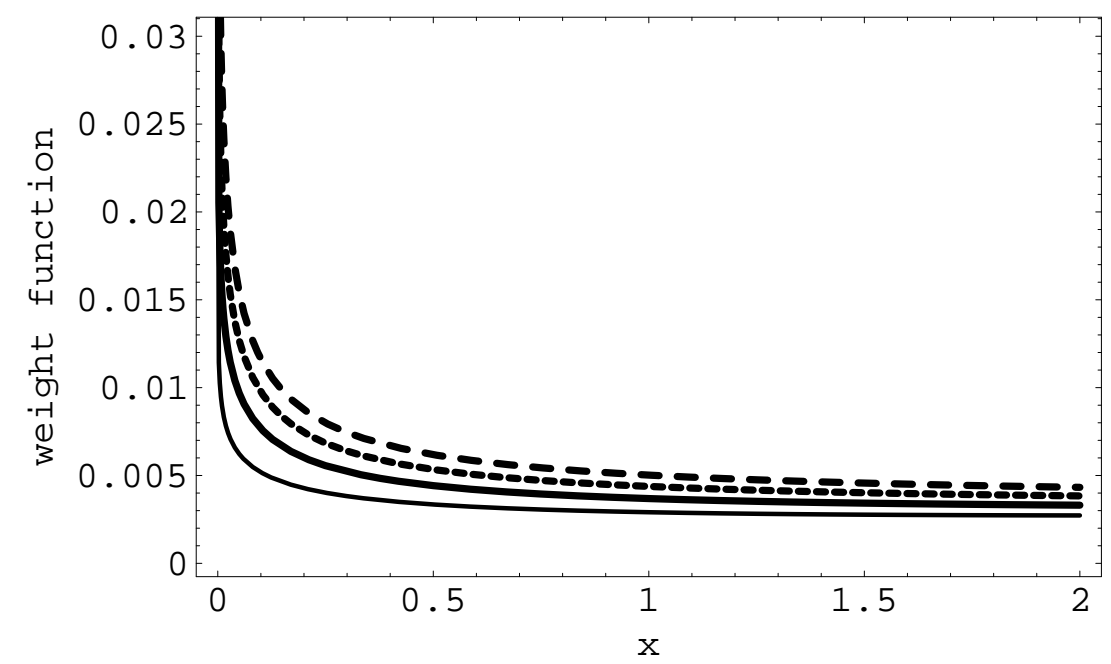

Figure 7. Plots of the weight function (161) of the GPAH-CS (157) versus $x=|z|^{2}$ with the parameters $m=3, \nu=2.8$ and for different values of the photon added number $p=1$ (thin solid line), $p=2$ (solid line), $p=3$ (dot line), and $p=4$ (dashed line).

\section{(ii) Overcompleteness}

The relation (48) gives in this case

$$
\int_{0}^{\infty} d x x^{n+p} g_{p}(x ; m)=\frac{\Gamma(m+\nu) \Gamma(n+1)^{2} \Gamma(n+2 m+2 \nu)^{2}}{\Gamma(2 m+2 \nu) \Gamma(n+p+1) \Gamma(n+m+\nu) \Gamma(n+p+2 m+2 \nu)}
$$

where the function $g_{p}$ is related to the measure $\omega_{p}$ by (85). Performing the variable change $n+m \rightarrow s-1$ in Eq. (158) gives:

$$
\int_{0}^{\infty} d x x^{s-1} h_{p}(x ; m)=\frac{\Gamma(s-p)^{2} \Gamma(s-p-1+2 m+2 \nu)^{2}}{\Gamma(s) \Gamma(s-p-1+m+\nu) \Gamma(s-1+2 m+2 \nu)}
$$

where $h_{p}(x ; m)=g_{p}(x ; m) \frac{\Gamma(2 m+2 \nu)}{\Gamma(m+\nu)}$. Application of the Mellin-inversion theorem (50) provides:

$$
h_{p}(x ; m)=G_{3,4}^{4,0}\left(\begin{array}{l|l}
x & \begin{array}{l}
0,-1+2 m+2 \nu,-p-1+m+\nu \\
-p,-p,-1-p+2 m+2 \nu,-1-p+2 m+2 \nu
\end{array}
\end{array}\right) .
$$

Then, the weight function is given by

$$
\begin{aligned}
\omega_{p}\left(|z|^{2} ; m\right)= & \frac{\Gamma(m+\nu)}{\pi} G_{3,4}^{1,3}\left(-|z|^{2} \mid \begin{array}{l}
-p, 1-2 m-p-2 \nu, 1-m-\nu \\
0,0,1-2 m-2 \nu, 1-2 m-\nu
\end{array}\right) \times \\
& \times G_{3,4}^{4,0}\left(|z|^{2} \mid \begin{array}{l}
p,-1+p+2 m+2 \nu,-1+m+\nu \\
0,0,-1+2 m+2 \nu,-1+2 m+2 \nu
\end{array}\right)
\end{aligned}
$$

where we have used (85), (155) and the multiplication formula of the Meijer's Gfunction (89). For $p=0$, the function $h_{p}$ is reduced to

$$
h_{0}\left(|z|^{2} ; m\right)=G_{1,2}^{2,0}\left(\begin{array}{l|l}
x & \begin{array}{l}
-1+m+\nu \\
0,-1+2 m+2 \nu
\end{array}
\end{array}\right)
$$

and the weight function becomes

$$
\omega_{0}\left(|z|^{2} ; m\right)=\frac{1}{\pi} G_{1,2}^{1,1}\left(\begin{array}{l|l}
-|z|^{2} & \begin{array}{l}
1-m-\nu \\
0,1-2 m-2 \nu
\end{array}
\end{array}\right) \cdot G_{1,2}^{2,0}\left(|z|^{2} \mid \begin{array}{l}
-1+m+\nu \\
0,-1+2 m+2 \nu
\end{array}\right) .
$$


In Figure 7, we plot the weight function (161) versus $x=|z|^{2}$ for $m=3, \nu=2.8$ and different values of the photon-added number $p=1,2,3,4$. All the curves are positive, this confirm the positivity of the weight function for the parameter $\nu>0$. As in the previous cases, the weight function presents a singularity at $x=0$ and tends to zero for $x \rightarrow \infty$.

(iii) Photon number statistics
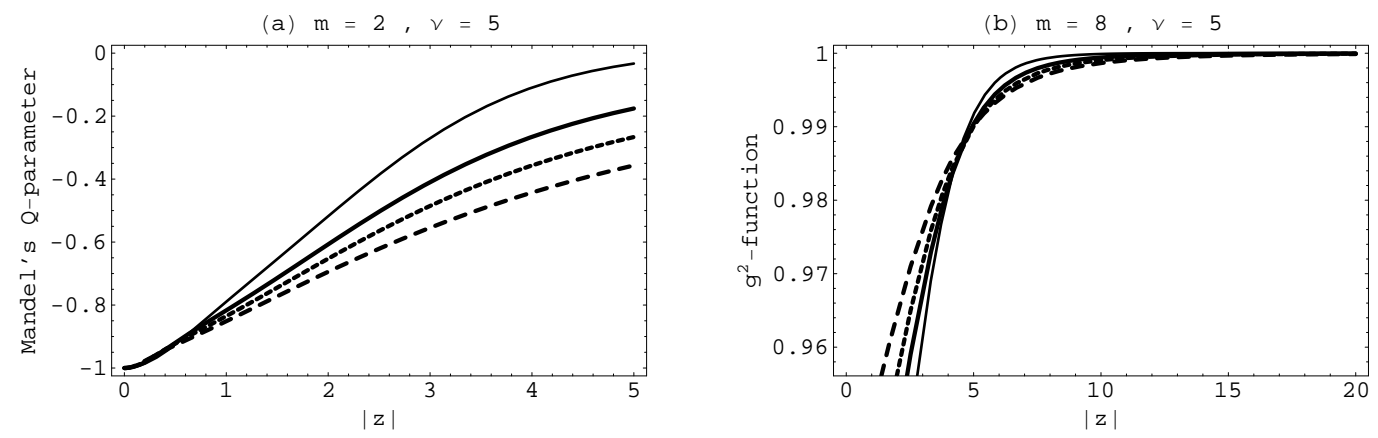

Figure 8. Plots of: (a) the Mandel Q-parameter (165) of the GPAH-CS (157) versus $|z|$ with the parameters $m=2$ and $\nu=5$ and for various values of the photon-added number $p$ with $p=1$ (thin solid line), $p=3$ (solid line), $p=5$ (dot line) and $p=8$ (dashed line); (b) Second-order correlation function (166) versus $|z|$, with the parameters $m=8$ and $\nu=5$ for various values of the photon-added number $p$ with $p=1$ (thin solid line), $p=3$ (solid line), $p=5$ (dot line) and $p=8$ (dashed line).
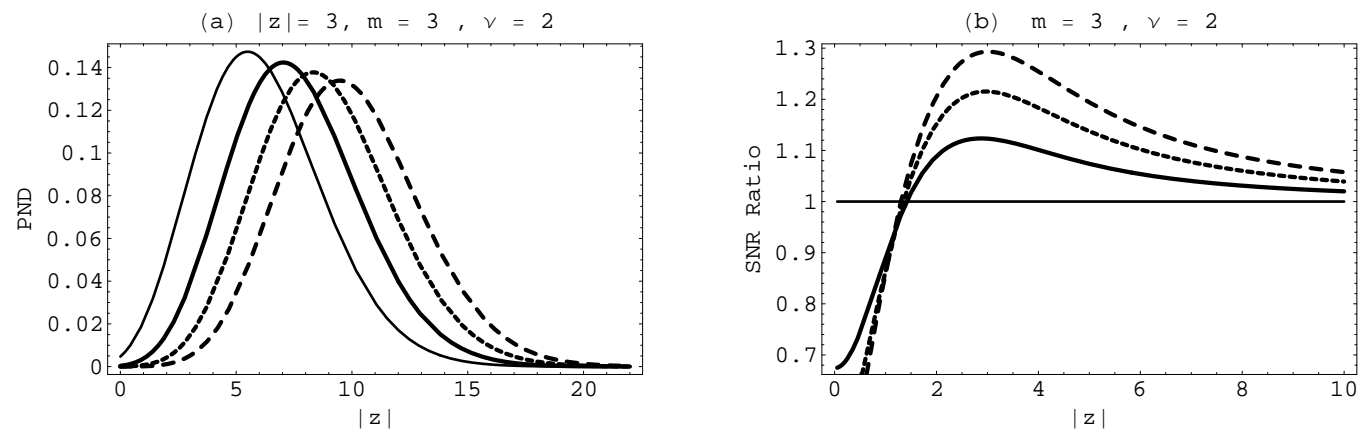

Figure 9. (a) Plot of the PND (167) of the GPAH-CS 157) versus the photon number $n$, with $|z|=3, m=3, \nu=2$ and for different values of the photon-added number $p$ with $p=0$ (thin solid line), $p=1$ (solid line), $p=2$ (dot line) and $p=3$ (dashed line).

(b) Plot of the SNR (169) ratios of GPAH-CS (157) to the corresponding GAH-CS versus $|z|$, with $m=3, \nu=2$ and for various values of the photon-added number $p$ with $p=0$ (thin solid line), $p=1$ (solid line), $p=2$ (dot line) and $p=3$ (dashed line)

Using the expressions (152) and (154) of the factors $K_{n}^{p}(m)$ and $\mathcal{N}_{p}\left(|z|^{2} ; m\right)$, respectively, we obtain the expectation values (62) and (63) as:

$$
\left\langle N_{m}\right\rangle=(m+p) \frac{{ }_{4} F_{4}\left(\begin{array}{ll}
1+p, m+p+1, p+2 m+2 \nu, m+\nu & ; \\
1, m+p, 2 m+2 \nu, 2 m+2 \nu & ;
\end{array}\right)}{{ }_{3} F_{3}\left(\begin{array}{ll}
1+p, 2 m+p+2 \nu, m \nu & ; \\
1,2 m+2 \nu, 2 m+2 \nu & ;
\end{array}\right)}
$$


Photon-added coherent states

$$
\left\langle N_{m}^{2}\right\rangle=(m+p)^{2} \frac{{ }_{5} F_{5}\left(\begin{array}{ll}
1+p, m+\nu, m+p+1, m+p+1, p+2 m+2 \nu & ; \\
1, m+p, m+p, 2 m+2 \nu, 2 m+2 \nu & ;|z|^{2}
\end{array}\right)}{{ }_{3} F_{3}\left(\begin{array}{ll}
1+p, 2 m+p+2 \nu, m \nu & ; \\
1,2 m+2 \nu, 2 m+2 \nu & ;
\end{array}\right)} .
$$

Then, the Mandel Q-parameter is reduced to:

$$
Q=(m+p)\left[\frac{{ }_{5} \mathcal{F}_{5}\left(|z|^{2} ; m, p\right)}{{ }_{4} \mathcal{F}_{4}\left(|z|^{2} ; m, p\right.}-\frac{{ }_{4} \mathcal{F}_{4}\left(|z|^{2} ; m, p\right)}{{ }_{3} \mathcal{F}_{3}\left(|z|^{2} ; m, p\right)}\right]-1 .
$$

The second order correlation function (60) follows as

$$
g^{2}={ }_{3} \mathcal{F}_{3}\left(|z|^{2} ; m, p\right) \frac{(m+p)_{5} \mathcal{F}_{5}\left(|z|^{2} ; m, p\right)-{ }_{4} \mathcal{F}_{4}\left(|z|^{2} ; m, p\right)}{(m+p){ }_{4} \mathcal{F}_{4}\left(|z|^{2} ; m, p\right)^{2}}
$$

where ${ }_{3} \mathcal{F}_{3},{ }_{4} \mathcal{F}_{4}$ and ${ }_{5} \mathcal{F}_{5}$ are the generalized hypergeometric functions:

$$
\begin{aligned}
& { }_{3} \mathcal{F}_{3}\left(|z|^{2} ; m, p\right)={ }_{3} F_{3}\left(\begin{array}{lll}
1+p, 2 m+p+2 \nu, m \nu & ; \\
1,2 m+2 \nu, 2 m+2 \nu & ; & |z|^{2}
\end{array}\right) \\
& { }_{4} \mathcal{F}_{4}\left(|z|^{2} ; m, p\right)={ }_{4} F_{4}\left(\begin{array}{ll}
1+p, m+p+1, p+2 m+2 \nu, m+\nu & ; \\
1, m+p, 2 m+2 \nu, 2 m+2 \nu & ; \quad|z|^{2}
\end{array}\right) \\
& { }_{5} \mathcal{F}_{5}\left(|z|^{2} ; m, p\right)={ }_{5} F_{5}\left(\begin{array}{ll}
1+p, m+\nu, m+p+1, m+p+1, p+2 m+2 \nu & ; \\
1, m+p, m+p, 2 m+2 \nu, 2 m+2 \nu & ; \quad|z|^{2}
\end{array}\right) .
\end{aligned}
$$

The PND (64) reads as

$\mathcal{P}_{n}^{p}\left(|z|^{2} ; m\right)=\frac{\Gamma(2 m+2 \nu)^{2} \Gamma(n+2 m+2 \nu) \Gamma(n+1) \Gamma(n-p+m+\nu)}{\Gamma(p+2 m+2 \nu) \Gamma(p+1) \Gamma(n-p+2 m+2 \nu)^{2} \Gamma(m+\nu)_{3} \mathcal{F}_{3}\left(|z|^{2} ; m, p\right)} \frac{|c z|^{2 n}}{(n !)^{2}}$.

For $p=0$, this reduces to PND of the conventional GAH-CS.

$\mathcal{P}_{n}^{p}\left(|z|^{2} ; m\right)=\frac{\Gamma(2 m+\mu) \Gamma(n+m+\nu)}{\Gamma(n+2 m+2 \nu) \Gamma(m+\nu){ }_{1} F_{1}\left(m+\nu ; 2 m+2 \nu ;|z|^{2}\right)} \frac{|c z|^{2 n}}{n !}$.

Finally, the SNR (65) gives

$$
\sigma^{p}\left(|z|^{2} ; m\right)=\frac{(m+p){ }_{4} \mathcal{F}_{4}\left(|z|^{2} ; m, p\right)-p_{3} F_{3}\left(1+p ; 1 ;|c z|^{2}\right)}{(m+p) \sqrt{{ }_{5} \mathcal{F}_{5}\left(|z|^{2} ; m, p\right)_{3} \mathcal{F}_{3}\left(|z|^{2} ; m, p\right)-\left({ }_{4} \mathcal{F}_{4}\left(|z|^{2} ; m, p\right)\right)^{2}}} .
$$

\section{(iv) Thermal statistics}

Starting with the normalized density operator expression (140), the $Q$-distribution or Husimi distribution analogue in the situation of the GPAH-CS (157) is provided as follows

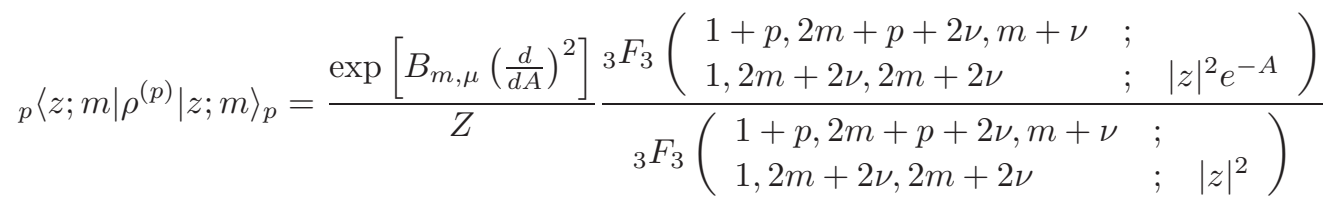

and in terms of Meijer's G functions,

$$
{ }_{p}\left\langle z ; m\left|\rho^{(p)}\right| z ; m\right\rangle_{p}=\frac{\exp \left[B_{m, \mu}\left(\frac{d}{d A}\right)^{2}\right]}{Z} \frac{G_{3,4}^{1,3}\left(-|z|^{2} e^{-A} \mid \begin{array}{l}
-p, 1-2 m-2 \nu-p, 1-m-\nu \\
0,0,1-2 m-2 \nu, 1-2 m-2 \nu
\end{array}\right)}{G_{3,4}^{1,3}\left(-|z|^{2} \mid \begin{array}{l}
-p, 1-2 m-2 \nu-p, 1-m-\nu \\
0,0,1-2 m-2 \nu, 1-2 m-2 \nu
\end{array}\right)} .
$$

After an angular integration, taking $x=|z|^{2}$, from the condition (68) we obtain

$$
\operatorname{Tr} \rho^{(p)}=\frac{\exp \left[B_{m, \mu}\left(\frac{d}{d A}\right)^{2}\right]}{Z} \int_{0}^{\infty} d x x^{p} G_{3,4}^{4,0}\left(x \mid \begin{array}{l}
0,-1+2 m+2 \nu,-1-p+m+\nu \\
-p,-p,-1-p+2 m+2 \nu,-1-p+2 m+2 \nu
\end{array}\right)
$$




$$
\times G_{3,4}^{1,3}\left(\begin{array}{l|l}
-x e^{-A} & \begin{array}{l}
-p, 1-2 m-2 \nu-p, 1-m-\nu \\
0,0,1-2 m-2 \nu, 1-2 m-2 \nu
\end{array}
\end{array}\right) .
$$

Applying the integral of Meijer's G-functions products properties to (172) gives the partition function expression provided in (143).

From the same diagonal elements in the states basis $|n+p\rangle$ as derived in (144), the quasi-distribution function $P\left(|z|^{2}\right)$ is determined through the relation

$$
\begin{aligned}
& \frac{1}{\bar{n}_{A}+1}\left(\frac{\bar{n}_{A}}{\bar{n}_{A}+1}\right)^{n} \frac{\Gamma(n+1)^{2} \Gamma(n+2 m+\mu)^{2}}{\Gamma(n+p+1) \Gamma(n+p+2 m+\mu)}=\int_{0}^{\infty} d x x^{n+p} P(x) \times \\
& G_{3,4}^{4,0}\left(x \mid \begin{array}{l}
0,-1+2 m+2 \nu,-1-p+m+\nu \\
-p,-p,-1-p+2 m+2 \nu,-1-p+2 m+2 \nu
\end{array}\right) .
\end{aligned}
$$

After performing the exponent change $n+p=s-1$ of $x=|z|^{2}$, in order to get the Stieltjes moment problem, we arrive at the $P$-function obtained as

$$
P\left(|z|^{2}\right)=\frac{1}{\bar{n}_{A}}\left(\frac{\bar{n}_{A}+1}{\bar{n}_{A}}\right)^{p} \frac{G_{3,4}^{4,0}\left(\frac{\bar{n}_{A}+1}{\bar{n}_{A}}|z|^{2} \mid \begin{array}{l}
0,-1+2 m+2 \nu,-1-p+m+\nu \\
-p,-p,-1-p+2 m+2 \nu,-1-p+2 m+2 \nu
\end{array}\right)}{G_{3,4}^{4,0}\left(|z|^{2} \mid \begin{array}{l}
0,-1+2 m+2 \nu,-1-p+m+\nu \\
-p,-p,-1-p+2 m+2 \nu,-1-p+2 m+2 \nu
\end{array}\right)} .
$$

satisfying the normalization to unity condition (70).

Then, the diagonal representation of the normalized density operator in terms of the GPAH-CS projector (69), takes the form

$\rho^{(p)}=\frac{1}{\bar{n}_{A}}\left(\frac{\bar{n}_{A}+1}{\bar{n}_{A}}\right)^{p} \int_{\mathbb{C}} d^{2} z \omega_{p}\left(|z|^{2} ; m\right)|z ; m\rangle_{p} \mathfrak{S}_{3,4}^{4,0}\left(|z|^{2}, \bar{n}_{A}\right)_{p}\langle z ; m|$

with $\mathfrak{S}_{3,4}^{4,0}\left(|z|^{2}, \bar{n}_{A}\right)$ the Meijer's G-functions quotient given in (174). From the relations (163), (164), and the definition (71), after computing the pseudo-thermal expectation values of the number operator and of its square, the thermal intensity correlation function (172) and the thermal analogue of the Mandel parameter (73) are obtained as above, with their expressions as in (107) and (108), respectively, with $\bar{n}_{A}$ instead of $\bar{n}_{o}$.

\subsection{Analysis of photon number statistics graphics}

In Figures 2, 5, 8, we represent the variations of (a) the Mandel Q-parameter 192, 132, 165), respectively, and (b) the second order correlation function (93, 133, 166), respectively, in terms of $|z|$,for different values of the photon-added number $p$ and for fixed values of the derivative order parameter $m$, and the polynomial parameter $\mu$ (or $\nu$ ). In these figures, the Mandel Q-parameter is strictly negative, increases with the amplitude $|z|$, and asymptotically tends to 0 . Besides, the second order correlation function is such that $0<g^{2}<1$ and asymptotically tends to 1 . Then, the GPAH-CS (80) exhibit subPoisonnian distribution, and get close to Poissonian distribution as $|z| \rightarrow \infty$.

In Figures 3, 6, 9 (a), the PND is ploted as a function of the photon number $n$, for a normal GAH-CS $p=0$ along with a GPAH-CS for different values $p=1,2,3$. It follows that the shift in the PND is more accentuated as the number of photons added increases. In Figures 2, 4, 6 (b), we plot the ratio of the SNR of various GPAH-CS, with $p=1,2,3$, to the SNR of a normal GAH-CS. The resulting curves reach their maximum over 1 
and asymptotically tends to 1, implying that the GPAH-CS SNR is above the ordinary GAH-CS SNR.

\section{Conclusion}

In this paper, a set of non-classical states, i.e, the generalized photon-added associated hypergeometric coherent states (GPAH-CS), have been considered. These states are obtained by repeated applications of the raising operator $a_{m}^{\dagger}$ on the generalized associated hypergeometric coherent states (GAH-CS). The required Klauder CS minimal set of conditions, i.e, the label continuity, the normalizability and the overcompleteness have been discussed. This latter property, evidenced through moment problems explicitly solved by using the Mellin inversion theorem in terms of the Meijer's G-function, has led to reproducing kernel analysis. Statistical quantities like the Mandel Q-parameter and the second-order correlation function have been derived from the number operator and its square expectation values. Besides, the signal-to-quantum-noise ratio (SNR), important for characterizing noiseless amplification of a coherent state, and also the photon number distribution (PND) evaluating the addition of photons for a given coherent light, have been analyzed. All these physical features have been depicted for appropriate values and discussed. In order to examine the thermal statistics of these states, the density matrix of a quantum canonical ideal gas of the system in thermodynamic equilibrium has been first given. Then, the Q-distribution or Husimi distribution, the diagonal representation of the density operator and the P-distribution function expressions in the GPAH-CS have been obtained. As interesting applications, the Hermite, Laguerre, Jacobi polynomials, and the hypergeometric functions have been studied. Their related classes of GPAH-CS as well the GAH-CS have been built, showing sub-Poissonian distribution, and their thermal statistics provided. For instance as $|z|$ takes large values, the Mandel parameter and the second-order correlation function tend to 0 and 1, respectively. Thus, the GPAH-CS become classical for large values of $|z|$. Moreover, increasing the number of photons leads to a shift of the PND.

\section{References}

[1] Schrödinger E 1926, Naturwiss 14664

[2] Glauber R J 1963 Phys. Rev. 1302529; 1963 Phys. Rev. 1312766

[3] Klauder J R 1963 J.Math. Phys. 41050

[4] Sudarshan E C G 1963, Phys. Rev. Lett. 10277

[5] Barut A O and Girardello L 1971 Commun. Math. Phys. 2141

[6] Perelomov A. M. 1972 Commun. Math. Phys. 26222

[7] Perelomov A M 1986 Generalized coherent states and their applications (Berlin: Springer)

[8] Ali S T, Antoine J P and Gazeau J P 2000, Coherent states, Waveletss and their generalizations, (New York : Springer)

[9] Ali S T, Antoine J-P, Gazeau J-P and Mueller U A Rev. Math. Phys. 7, 19951013

[10] Klauder J R, Skagerdtam B-S 1985 Coherent states: applications in physics and mathematical physics, (Singapore : World Sientific)

[11] Gazeau J - P 2009 Coherent states in quantum mechanics (Weinheim : Wiley-VCH) 
[12] Brif C and Mann A 1988 J. Phys. A: Math. Gen. 31 L9

[13] Vourdas A 1997 J. Phys. A: Math. Gen. 304867

[14] Berezin F A 1986 The method of Second Quantization (Moscow: Nauka)

[15] Aremua I, Gazeau J-P and Hounkonnou M N 2012 J. Phys. A: Math. Gen 45335302

[16] Aremua I, Hounkonnou M N and E. Baloïtcha 2015 Rep. Math. Phys. 45(2) 247

[17] Aragone C, Guerri G, Salamó S and Tani J L 1974 J. Phys. A: Math. Nucl. Gen. 7 L149

[18] Nieto M M and Simmons L M Jr 1978 Phys. Rev. Lett. 41207

[19] Cotfas N 2002 J. Phys. A: Math. Gen. 359355

[20] Aleixo A N F, Balantekin A B and Cândido Ribeiro M A 2002 J. Phys. A: Math. Gen. 359063

[21] Hounkonnou M N and Sodoga K 2005 J. Phys. A: Math. Gen 387851

[22] Agarwal G S and Tara K 1991 Phys. Rev A. bf 43492 ; 1992 Phys. Rev A. bf 46485

[23] Berrada K 2015 J. Math. Phys 56 072104; Li Y, Jing H and Zhan M-S 2006 J. Phys. B 392107 ; Berrada K, Abdel-Khalek S, Eleuch H and Hassouni Y 2013 Quant. Inf. Process. 1269.

[24] Dodonov V V Korenmoy Ya A Man'ko V I and Moukhin Y A 1996 Quantum Semiclass. Opt. 8 413

[25] Sixderniers and Penson K A 2001 J. Phys. A. Math. Gen 342859

[26] Klauder J R Penson K A and Sixderniers J-M 2001 Phys. Rev. A 64013817

[27] Popov D 2002 J. Phys. Math. Gen 357205

[28] Popov D Zaharie I and Dong S H 2006 Czech. J. Phys. 56 157; Popov D 2006 Electron. J. Theor. Phys. (EJTP) 3(11) 123

[29] Hounkonnou M N and Ngompe Nkouankam E B 2008 J. Phys A: Math. Theor. 42(2)

[30] Mojaveri B Dehghani A and Mahmoodi S 2014 Phys. Scr. 89085202

[31] Daoud M 2002 Phys. Lett. A. 305135

[32] Appl T and Schiller D H 2004 J. Phys. A: Math. and Gen. 372731

[33] Welsch D-G Dakna M Knöll L and T. Opatrny, Los Ala- mos e-print quant-ph/9708018.

[34] Dakna M Anhut T Opatrny T Knöll L and Welsch D-G 1997 Phys. Rev.A. 553184 Dakna M Knöll L and Welsch D-G 1998 Opt. Commun. 145309

[35] Ban M 1996 J. Mod. Opt. 431281

[36] Mandel L and Wolf E 1995 Optical coherence and quantum optics (Cambridge: Cambridge University Press)

[37] Penson K A and Solomon A I 1999 J. Math. Phys. 402354

[38] Marichev O I 1983 Handbook of integral transforms of higher transcendental functions: theory and algorithmic tables (Chichester, UK: Ellis Harwood)

[39] Prudnikov A P, Brychkov Y A and Marichev O I 1998 Integrals and series (new York: Gordon and Breach)

[40] Gard B T Li D You C Seshadreesan K P Birrittella R Luine J Rafsanjani S M H Mirhosseini M Omar Magaña-Loaiza O S Koltenbah B E Parazzoli C G Capron B A Boyd R W Gerry C C Lee H and Dowling J P arXiv:1303.3682 [quant-ph] (2013)

[41] A. M. Mathai and R. K. Saxena 1973 Generalized Hypergeometric Functions with Applications in Statistics and Physical Sciences (Lecture Notes in Mathematics vol 348) (Berlin: Springer) 